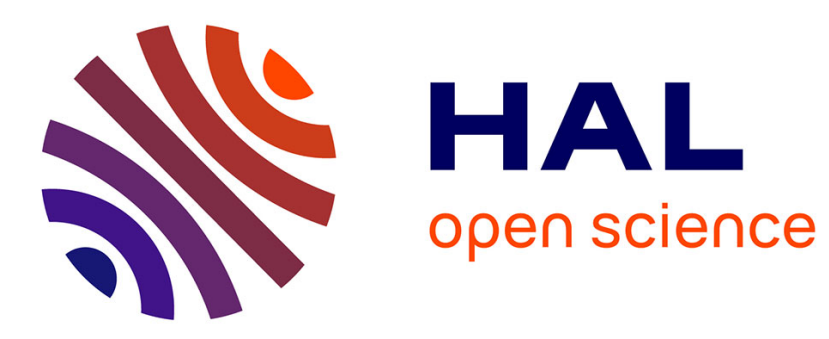

\title{
A virtual chemical mechanism for prediction of NO emissions from flames
}

Giampaolo Maio, Mélody Cailler, Alberto Cuoci, Benoît Fiorina

\section{To cite this version:}

Giampaolo Maio, Mélody Cailler, Alberto Cuoci, Benoît Fiorina. A virtual chemical mechanism for prediction of NO emissions from flames. Combustion Theory and Modelling, 2020, 24 (5), pp.872-902. 10.1080/13647830.2020.1772509 . hal-03419970

\section{HAL Id: hal-03419970 \\ https://hal.science/hal-03419970}

Submitted on 8 Nov 2021

HAL is a multi-disciplinary open access archive for the deposit and dissemination of scientific research documents, whether they are published or not. The documents may come from teaching and research institutions in France or abroad, or from public or private research centers.
L'archive ouverte pluridisciplinaire HAL, est destinée au dépôt et à la diffusion de documents scientifiques de niveau recherche, publiés ou non, émanant des établissements d'enseignement et de recherche français ou étrangers, des laboratoires publics ou privés. 


\title{
RESEARCH ARTICLE
}

\section{A virtual chemical mechanism for prediction of $\mathrm{NO}$ emissions from flames}

\author{
G. Maio ${ }^{\mathrm{a}}$, M. Cailler ${ }^{\mathrm{b}}$, A. Cuoci $^{\mathrm{c}}$ and B. Fiorina ${ }^{\mathrm{a}}$ \\ a Laboratoire EM2C, CNRS, CentraleSupélec, Université Paris-Saclay, 3 rue Joliot Curie \\ 91192 Gif Sur Yvette cedex, France \\ bSAFRAN Tech, Rue des Jeunes Bois, Châteaufort - CS 80112, 78772 Magny-les-Hameaux, \\ France \\ ${ }^{c}$ Department of Chemistry, Materials and Chemical Engineering "G. Natta", Politecnico di \\ Milano, Milano 20133, Italy
}

\author{
ARTICLE HISTORY \\ Compiled May 17, 2020
}

\begin{abstract}
A reduced order kinetic model for NO (Nitric Oxide) prediction, based on the virtual chemistry methodology [1], is developed and applied. Virtual chemistry aims to optimize thermochemical properties and kinetic rate parameters of a network of virtual species and reactions. A virtual main chemical mechanism is dedicated to temperature and heat release prediction and is coupled with the flow governing equations, whereas satellite sub-mechanisms are designed to predict pollutants formation. Two virtual chemistry mechanisms are here employed: a main mechanism for calculating the temperature and heat release rate and a second mechanism dedicated to NO prediction. To recover the chemical structure of multi-mode combustion, both premixed and non-premixed flamelets are included in the learning database used to optimize the virtual NO mechanism. A multi-zone optimization procedure is developed to accurately capture both fast and slow NO chemistry that include prompt, thermal and reburning pathways. The proposed NO sub-mechanism and optimization methodology are applied to $\mathrm{CH}_{4}$ /air combustion. Laminar 1-D premixed and non-premixed flamelet configurations are first tested. The approach is then further assessed in 2-D CFD laminar flame simulations, by providing a direct comparison against detailed chemistry. 2-D premixed, non-premixed and partially premixed flame configurations are numerically investigated. For all cases, the virtual mechanism fairly captures temperature and $\mathrm{NO}_{x}$ chemistry with only 12 virtual species and 8 virtual reactions with a drastic CPU time reduction compared to detailed chemistry.
\end{abstract}

\section{KEYWORDS}

Nitric oxide ; virtual chemistry ; laminar flames ; reduced chemistry

\section{1. Introduction}

2 Nitrogen oxides $\left(\mathrm{NO}_{x}\right)$ emitted in the atmosphere, even in small quantities, cause prob-

3 lems to the local air quality. They contribute to acid rain, ozone production and smog

4 formation. Combustion processes are the main source of nitrogen oxides emissions $5[2]$. To limit their production engineers need numerical tools to design and optimize

CONTACT B. Fiorina. Email: benoit.fiorina@centralesupelec.fr 



Figure 1.: Temperature and $\mathrm{NO}$ mass fraction profiles from $\mathrm{CH}_{4}$ /air 1-D premixed flames computed at two different equivalence ratios (stoichiometric and rich) with the GRI3.0 mechanism [3]. Pressure is equal to 1 atm and the initial temperature equals 300K. The computational domain covers $1 \mathrm{~m}$ and includes the flame front and postflame regions. The inner zoom plots show the NO mass fraction with a domain length comparable with the flame thermal thickness.

combustion devices. The numerical prediction of $\mathrm{NO}_{x}$ emission is a challenging task for three main reasons: $i$ ) $\mathrm{NO}_{x}$ are produced in very small quantities; ii) $\mathrm{NO}_{x}$ formation and consumption feature multiple chemical time scales and iii) $\mathrm{NO}_{x}$ chemical paths vary with the operating conditions (fuel, temperature, pressure, equivalence ratio, etc.). At the combustion chamber exit, $\mathrm{NO}_{x}$ are mainly composed of $\mathrm{NO}$ [4], whose chemistry complexity is well illustrated in Fig. 1. The detailed chemistry solutions of stoichiometric and rich $(\phi=1.6)$ premixed 1-D freely propagating laminar flames, obtained using REGATH solver [5] and GRI3.0 detailed mechanism [3] for $\mathrm{CH}_{4} /$ air combustion, are shown. A thin flame front region zone, of the order of the millimeter, is first identified within the thermal flame thickness. NO chemistry has here a characteristic time scale comparable to the fuel oxidation process. The chemical pathway, leading to this flame front NO formation, is identified as prompt route [6, 7]. A second zone is observed in the post-flame region where temperature and major species reach chemical equilibrium, while NO mass fraction still evolves slowly. Furthermore, in the post-flame zone, NO chemistry exhibits two different behaviours:

- For lean, stoichiometric and moderately rich conditions: a slow, monotonic, NO production is observed until the chemical equilibrium is reached. This process is mainly governed by the thermal [8] route.

- For very rich conditions $(\phi \geq 1.4)$ : the slow NO formation competes with NO recombination [9] causing a non-monotonic evolution of NO mass fraction. The NO consumption is known as reburning process [10, 11].

Detailed chemistry mechanisms currently include and combine prompt, thermal and reburning pathways, to describe $\mathrm{NO}$ formation in as many as possible flame conditions [12]. However hydrocarbons detailed chemical mechanisms involve hundreds of species 
and reactions [13] and their size further increase with the consideration of NO chemistry. For example in the GRI3.0 mechanism [3], widely used to describe $\mathrm{CH}_{4}$ oxidation, the $\mathrm{NO}_{x}$ chemistry subset adds 17 species and 108 reactions to the initial mechanism made of 36 species and 217 reactions. As direct inclusion of detailed chemistry in CFD simulations causes CPU cost issues, reduced order models are needed to mitigate the computational burden $[14,13,15]$. Three main modeling strategies are currently employed in the literature to model combustion chemistry at reduced CPU cost $[15,16]$ : global mechanisms $[17,18]$, tabulated chemistry $[19,20,21]$ and analytically reduced chemistry $[22,23]$.

A widely used technique to capture main combustion chemistry properties is to develop and optimize empirical global and semi-global mechanisms [24, 25], containing from 1 to 4 reaction steps. Unfortunately these mechanisms are not suitable to predict NO because, including only a few number of species, they do not contain NO chemistry species.

An alternative strategy is the systematic reduction of detailed mechanisms. Analytically reduced mechanisms [13] have been especially developed to capture NO formation [26]. This strategy allows to predict NO formation [22] over multiple flame regimes with an acceptable error. However, the application of analytically reduced mechanism to large scale simulations $[26,27]$ is still CPU demanding.

The last standard route for chemistry reduction is the "tabulated chemistry" formalism $[19,28]$. It aims at pre-computing, in a preliminary step, the reduced manifold in which the chemical subspace evolves. The manifold is finally coupled with a CFD solver to model the combustion process. In flamelet based tabulated chemistry methods, all detailed chemistry ingredients are included within a chemical table build-up from a collection of $0-\mathrm{D}$ reactors or $1-\mathrm{D}$ flame archetypes [20, 21, 29]. The definition and the coordinates of the chemical database depend on the complexity of the targeted computed flame regime. In the literature, the potential of tabulated chemistry for NO formation prediction has been investigated first by Nafe and Mass [30]. Analysing PSRs (Perfectly Stirred Reactors) solutions, using the ILDM (Intrinsic LowDimensional Manifolds) [28] approach, they conclude that additional slow time scales associated with NO chemistry exist. In the flamelet framework one strategy, to model $\mathrm{NO}$ formation, is to include nitrogen species in the progress variable definition and to add a dedicated transport equation for NO mass fraction in the flow solver [31, 32]. Another strategy, originally developed in the FPV formalism (flamelet/progress variable) [33] and then adapted to the FGM model (flamelet generated manifolds) [34], consists in splitting the NO chemical source term in a production and a consumption contributions and in adding an additional transport equation for the NO mass fraction in the flow solver. An original tabulated chemistry approach, called NOMANI model, has been proposed by Pecquery et al. in the FPI (Flame prolongation of ILDM) context [35]. In the NOMANI model, the NO source term is split in a flame front contribution and in a burnt gases contribution which are extracted from two separate look-up tables (carbon and nitrogen), parametrised from two different progress variables. The NORA model (NO Relaxation Approach) [36], dedicated to thermal NO prediction in internal combustion engines, is another example of tabulated chemistry application to NO prediction using a collection of PSRs solutions. However particular attention is required when NO mass fraction is retained as a progress variable in the post-flame region. As shown in Fig 1b, in rich conditions, NO mass fraction is not strictly monotonous and cannot be considered as progress variable. Godel et al. [31] overcame this limitation, by using an optimized combination of N-species to build-up an appropriate NO chemistry progress variable valid also for rich conditions. 


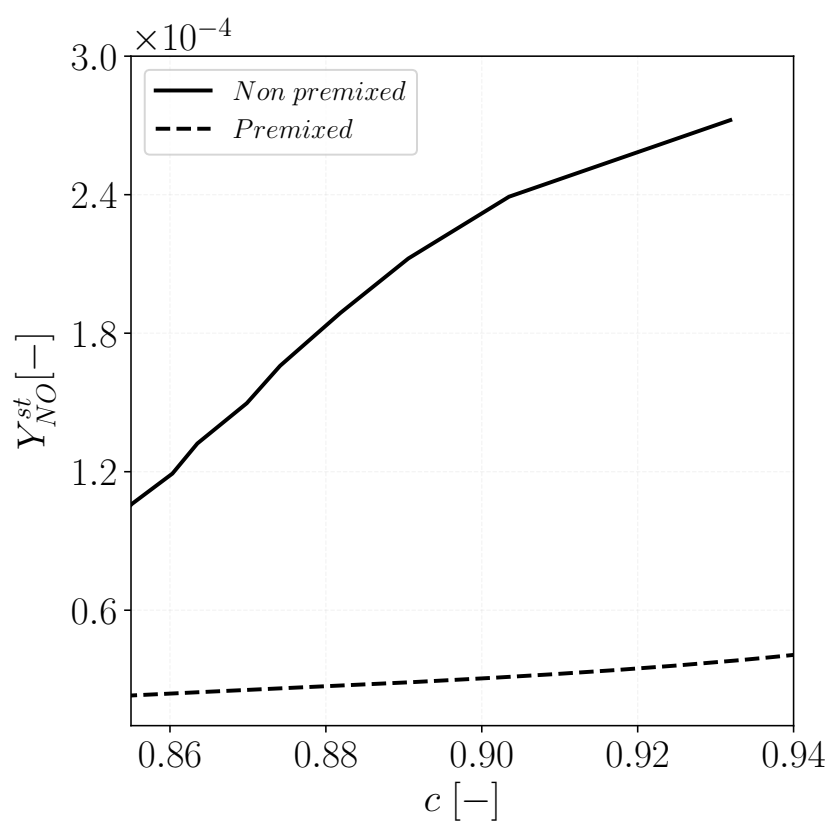

Figure 2.: NO mass fraction flamelet trajectories for a $\mathrm{CH}_{4} /$ air mixture, at atmospheric pressure and initial temperature of 300K, computed with GRI3.0 mechanism [3]. The data are plotted as a function of progress variable (c) over the stoichiometric mixture fraction $\left(\mathrm{Z}_{s t}\right)$ cut-plane. Data are extracted from a premixed and a non-premixed database.

Despite the extensive and successful applications, tabulated chemistry fails when the local flame structure differs from the tabulated archetype [37, 38, 39]. As an example, Fig. 2 shows the trajectories projection in the $\mathrm{Y}_{N O}$-progress variable (c) sub-space over the stoichiometric cut-plane $\left(\mathrm{Z}=\mathrm{Z}_{s t}\right)$, of both premixed and non-premixed 1-D flamelets. The 1-D flames are computed using REGATH solver [5] and GRI3.0 detailed mechanism [3] for $\mathrm{CH}_{4}$ /air mixture. The $\mathrm{NO}$ mass fraction trajectories show significant differences (up to an order of magnitude) between the two databases for the same mixture fraction $\left(\mathrm{Z}_{s t}\right)$ and the same progress variable $(\mathrm{c})$ values. This demonstrates that tabulated chemistry, does not perform well for NO formation in multi-mode combustion, if a single flame regime is accounted in the database generation.

An alternative reduction chemistry route, named virtual chemistry, has been recently developed by Cailler et al. [40]. The method consists in building-up empirical mechanisms made of virtual species and reactions. As in tabulated chemistry, an ensemble of reference flame archetypes is first computed. However instead of "tabulating" low-dimensional manifolds, thermodynamic and chemical properties of the virtual components are optimized to fit an ensemble of targeted flame solutions. It has been observed by Cailler et al. [1] that multi-mode combustion regimes are well captured with a limited number of virtual species and virtual reactions as soon as both premixed and non-premixed flame elements are included in the learning database. CO emissions have been accurately predicted in a turbulent confined aeronautical combustor exposed to heat losses [41]. Virtual chemistry is then a good candidate to model NO formation in hybrid flame structures.

The objective of the present study is to propose a new reduced mechanism in the 
virtual chemistry formalism able to predict NO in hybrid combustion regimes. The challenge is to account for all the NO chemistry pathways, included in detailed chemistry for premixed and non-premixed flames. In section 2 , the NO mechanism is introduced whereas the optimization strategy and the application to $\mathrm{CH}_{4}$ /air combustion are described in section 3. Section 4 shows and discusses the validation results in 1-D premixed freely propagating flames and in non-premixed counterflow flames, comparing the virtual chemistry results against simulations carried out with the detailed kinetic mechanism. In section 5, the proposed mechanism is further assessed in 2-D CFD laminar flame simulations, employing the open source solver laminarSMOKE [42]. A premixed, a non-premixed and a partially premixed flame configuration are studied.

\section{Virtual mechanism architecture}

\subsection{Virtual chemistry concept}

A virtual chemical scheme is made of an ensemble of optimized mechanisms dedicated to predict user-defined flame properties. Quantities of interest might be the heat release and the temperature [40] or the formation of some pollutants such as for instance carbon monoxide $[1,41]$ or nitrogen oxides.

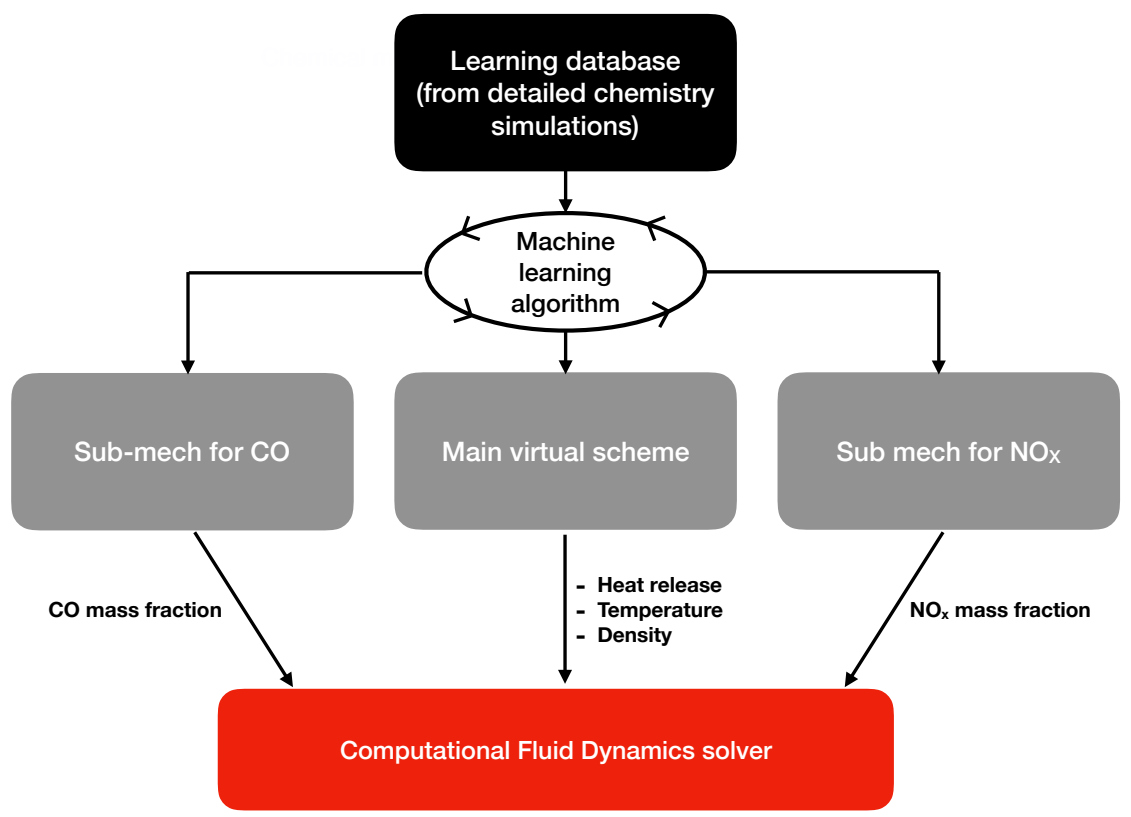

Figure 3.: Schematic representation of the virtual chemistry methodology. In this example the virtual scheme is made of a main mechanism dedicated to predict the heat release, temperature and density and two sub-mechanisms designed to model the formation of $\mathrm{CO}$ and $\mathrm{NO}_{X}$, respectively.

The general methodology to design and optimize a virtual chemical scheme is schematized in Fig. 3. A learning database is first built-up from detailed chemistry solutions of canonical flame archetypes. A main mechanism composed of virtual species and reactions is then designed and trained through a machine learning algorithm to target the thermal flame structures given by the learning database. More precisely, the main 
virtual scheme models the reactive mixture thermodynamic and transport properties as well as the heat released by combustion. The main mechanism is then implemented in a CFD flow solver through the mass, momentum and energy equations.

Detailed chemistry ingredients are accounted for during the optimization stage of the main mechanism but, as integrated flame quantities (flame speed, heat release, etc.) and temperature are targeted, individual species information are not accessible anymore. Independent satellite virtual sub-mechanisms are designed and optimized to access specific species mass fractions of interest. As an example, a virtual submechanism dedicated to CO prediction has been developed in [1] and applied to a turbulent flame LES in [41].

This section proposes a virtual chemical scheme architecture for NO prediction. The virtual chemistry mathematical formalism is first presented in section 2.2 and a main virtual mechanism adequate for hydrocarbon-air combustion is then reviewed in section 2.3. A NO virtual sub-mechanism is proposed and discussed in section 2.4.

\subsection{Virtual chemistry formalism}

A virtual scheme $X$ includes $N_{s}$ virtual species and $N_{r}$ virtual reactions, where $\mathrm{X}$ denotes either the main mechanism or a sub-mechanism. Any virtual mechanism $X$ is composed by an ensemble of reversible virtual reactions $R_{i}^{X}$ :

$$
\sum_{k=1}^{N_{s}^{i}} \alpha_{k i}^{\prime} \nu_{k} \leftrightarrow \sum_{k=1}^{N_{s}^{i}} \alpha_{k i}^{\prime \prime} \nu_{k}
$$

where $N_{s}^{i}$ is the number of virtual species involved in the virtual reaction $R_{i}^{X} . \nu_{k}$ denotes the $k^{t h}$ virtual species whereas $\alpha_{k i}^{\prime}$ and $\alpha_{k i}^{\prime \prime}$ are the reactant and product mass stoichiometric coefficients, respectively. The reaction progress $q_{i}$, for the reaction $R_{i}^{X}$, is closed using a finite rate formulation with modified reaction orders:

$$
q_{i}=k_{f i} \prod_{k=1}^{N_{s}}\left[\nu_{k}\right]^{F_{k}^{i}}-k_{b i} \prod_{k=1}^{N_{s}}\left[\nu_{k}\right]^{B_{k}^{i}}
$$

where $\left[\nu_{k}\right]$ is the $k^{t h}$ molar species concentration, $F_{k}^{i}$ and $B_{k}^{i}$ are the forward and backward reaction orders corresponding to the $k^{t h}$ species in the $i^{t h}$ reaction. $k_{f i}$ and $k_{b i}$ are the forward and backward rate constants. $k_{f i}$ is expressed using an Arrheniuslike formulation:

$$
k_{f i}=A_{i} T^{\beta_{i}} \exp \left(\frac{-E_{a}^{i}}{R T}\right)
$$

where $A_{i}$ is the pre-exponential factor, $E_{a}^{i}$ is the activation energy and in the modified Arrhenius law a temperature exponent $\beta_{i}$ is added. When $R_{i}^{X}$ is a reversible reaction, the backward rate constant is related to the forward one through the following relation:

$$
k_{b i}=\frac{k_{f i}}{K_{e q, i}}
$$


with $K_{e q, i}$ the equilibrium constant of reaction $R_{i}^{X}$ defined as:

$$
K_{e q, i}=\prod_{k=1}^{N_{s}^{i}}\left(\left[\nu_{k}\right]||^{e q}\right)^{\alpha_{k i}^{\prime \prime}-\alpha_{k i}^{\prime}}
$$

where $\left.\left[\nu_{k}\right]\right|^{e q}$ is the targeted equilibrium concentration of species $\nu_{k}$.

The kinetic rate parameters of virtual reactions in the virtual mechanism $X$ are optimized through the evolutionary algorithm proposed by Cailler et al. [40]. The optimization problem consists in minimizing the following cost function $\mathrm{C}$ which aims to compare the virtual and detailed chemistry solutions through a linear combination of selected normalized flame quantities:

$$
C=f\left(\mathbf{w}^{v}\left(\chi^{\mathbf{v}}\right), \mathbf{w}^{d}\left(\chi^{\mathbf{d}}\right)\right)
$$

where the vector $\mathbf{w}=\left(\rho u, \rho v, \rho w, \rho Y_{k}, T\right)$ is the solution of the system of flow governing equations, which depends on the set of parameters $\chi$. The vector $\chi$ includes the set of thermodynamic, transport and kinetic rate parameters. ${ }^{d}$ and ${ }^{v}$ superscripts refer to detailed and virtual chemistry, respectively. $\chi^{\mathrm{d}}$ is given by the complex thermodynamic and transport properties and by the detailed chemical scheme involved to build-up the learning database. $\chi^{\mathbf{v}}$ is the set of thermo-chemistry and transport parameters to optimize, which constitutes the output of the optimization procedure.

\subsection{Main mechanism}

Different forms of virtual mechanism have been tested to recover the thermal flame structure of a learning database composed of both premixed and non-premixed flamelets. Tests performed in [40] showed that one-step chemical schemes does not have enough degrees of freedom to handle both fast exothermic and slow endothermic $\mathrm{CO}$ reactions observed under rich conditions. On the contrary a virtual mechanism made of two consecutive reactions accurately capture the thermal flame structure of both premixed and diffusion flames. The following 2-step main virtual mechanism is therefore retained:

$$
\begin{aligned}
& \alpha_{F}^{T, 1} F+\alpha_{O x}^{T, 1} O x \rightarrow I \\
& I \rightarrow \sum_{k=1}^{N_{P}^{v}} \alpha_{P_{k}}^{T, 2} P_{k}
\end{aligned}
$$

where $\alpha_{k}^{X, i}$ are stoichiometric coefficients per mass unit. Fuel (F) and oxidizer (Ox) are transformed through reaction $R_{1}^{T}$ in an intermediate species (I) which is then converted by reaction $R_{2}^{T}$ into $N_{P}^{v}$ virtual products $\mathrm{P}_{k}$. Extensive tests conducted in [40] showed that an accurate prediction of equilibrium flame temperature over the whole range of equivalence ratio is obtained with $N_{P}^{v}=4$.

The reaction progresses of the two irreversible reactions $R_{1}^{T}$ and $R_{2}^{T}$ are closed with extended Arrhenius formulation as follows: 


$$
\begin{aligned}
& q_{1}^{T}=A_{1}^{T}(Z) \exp \left(\frac{-E_{a}^{T, 1}}{R T}\right)[F]^{F_{F}^{T, 1}}[O]^{F_{O x}^{T, 1}} \\
& q_{2}^{T}=A_{2}^{T} \exp \left(\frac{-E_{a}^{T, 2}}{R T}\right)[I]^{F_{I}^{T, 2}(Z)}
\end{aligned}
$$

Contrary to standard Arrhenius formulations, the pre-exponential constant of Eq. 6 and the reaction order $F_{I}^{T, 2}(Z)$ are expressed as dependent on the mixture fraction $Z$, and calibrated to match the laminar flame speed and thickness within the flammability limits [40]. As preferential species diffusion is not accounted for, the mixture fraction is given by the solution of an additional passive scalar balance equation [15].

Thermodynamic and transport properties of the virtual species as well as specific gas constants are calibrated to recover averaged properties of the multi-component real mixture. In addition, equilibrium constants are defined to retrieve real equilibrium compositions. For for the sake of conciseness, details of virtual species thermodynamics properties are not given here, but we encourage the reader to refer to [1] for further details. The next section is dedicated to the description of the virtual NO sub-mechanism, which constitutes the main novelty of this work.

\subsection{NO mechanism}

A mechanism architecture is proposed to account for the whole NO chemical pathways evidenced previously in Fig. 1: prompt, thermal and reburning. For that purpose, the following NO virtual sub-mechanism, composed of 6 reactions, is proposed:

$$
\begin{aligned}
& \alpha_{F}^{N O, 1} F+\alpha_{O x}^{N O, 1} O x \rightarrow \alpha_{V_{1}}^{N O, 1} V_{1}+\alpha_{V_{2}}^{N O, 1} V_{2}+\alpha_{V_{3}}^{N O, 1} V_{3} \quad\left(R_{1}^{N O}\right) \\
& V_{1}+F+O x \rightarrow \alpha_{N O}^{N O, 2} N O+\alpha_{V_{2}}^{N O, 2} V_{2}+F+O x \quad\left(R_{2}^{N O}\right) \\
& \mathrm{F}+\mathrm{NO} \rightarrow \mathrm{F}+\mathrm{V}_{2} \quad\left(R_{3}^{N O}\right) \\
& V_{3} \rightarrow N O \quad\left(R_{4}^{N O}\right) \\
& V_{3} \rightarrow V_{2} \quad\left(R_{5}^{N O}\right) \\
& V_{2} \leftrightarrow N O \quad\left(R_{6}^{N O}\right)
\end{aligned}
$$

where $\alpha_{k}^{N O, i}$ are the mass stoichiometric coefficients associated to species $k$ in the reaction $R_{i}$. An ensemble of virtual species $V_{1}, V_{2}$ and $V_{3}$ is first produced through the initiation reaction $R_{1}^{N O}$. Note that unlike to "real" mechanism, element conservation is not required in virtual reactions as intermediate species $V_{1}, V_{2}$ and $V_{3}$ does not have any physical representation, but represent degrees of freedom of the model.

Reactions $R_{2}^{N O}$ and $R_{3}^{N O}$ are dedicated to model the fast NO chemistry, which is dominant at the flame front scale. $R_{3}^{N O}$ is designed to reproduce the fast NO reburning that is especially relevant when an excess of hydrocarbon radicals is present in the system [11]. In addition, as it will be shown in section 4.3 , using two reactions $\left(R_{2}^{N O}\right.$ $R_{3}^{N O}$ ) to model fast NO formation, instead of one $\left(R_{2}^{N O}\right)$, enables a better prediction of both premixed and non-premixed NO profiles. Virtual reactions $R_{4}^{N O}$ to $R_{6}^{N O}$ describe the NO formation associated with slow post-flame chemistry. It includes thermal NO 
pathway and slow NO reburning phenomena. As discussed later in section 4.2, the combination of three reactions allows an efficient description of slow NO post-flame pathways in both lean and rich regimes. Finally, the equilibrium reaction $R_{6}^{N O}$ ensures that the chemical equilibrium conditions are well retrieved.

The rate of progress for the reactions set $R_{1}^{N O}-R_{6}^{N O}$ are closed using the following Arrhenius-like expressions:

$$
\begin{aligned}
& q_{1}^{N O}=A_{1}^{N O}(Z) \exp \left(\frac{-E_{a}^{N O, 1}}{R T}\right)[F]^{F_{F}^{N O, 1}}[O]^{F_{O x}^{N O, 1}} \\
& q_{2}^{N O}=A_{2}^{N O}(Z) \exp \left(\frac{-E_{a}^{N O, 2}}{R T}\right) T_{T}^{\beta_{T}^{N O, 2}}\left[V_{1}\right]^{F_{V_{1}}^{N O, 2}}[F][O x] \\
& q_{3}^{N O}=A_{3}^{N O} \exp \left(\frac{-E_{a}^{N O, 3}}{R T}\right)[F][N O]^{F_{N O}^{N O, 3}} \\
& q_{4}^{N O}=A_{4}^{N O}(Z) \exp \left(\frac{-E_{a}^{N O, 4}}{R T}\right)\left[V_{3}\right]_{V_{3}}^{F^{N O, 4}} \\
& q_{5}^{N O}=A_{5}^{N O}(Z) \exp \left(\frac{-E_{a}^{N O, 5}}{R T}\right)\left[V_{3}\right]_{V_{3}}^{F^{N O, 5}} \\
& q_{6}^{N O}=A_{6}^{N O}(Z) \exp \left(\frac{-E_{a}^{N O, 6}}{R T}\right)\left([N O]^{F_{N O}^{N O, 6}}\left[V_{2}\right]^{F_{V_{2}}^{N O, 6}}-\frac{[N O]^{B_{N O}^{N O, 6}}\left[V_{2}\right]^{B_{V_{2}}^{N O, 6}}}{K_{c}^{N O, 6}}\right)
\end{aligned}
$$

The equilibrium constant for the reversible reaction $R_{6}^{N O}$ is computed as follows:

$$
K_{c}^{N O, 6}=\frac{\left.[N O]^{d}\right|^{e q}}{\left.\left[V_{2}\right]^{v}\right|^{e q}}
$$

where $\left.[N O]^{d}\right|^{e q}$ is the equilibrium NO molar concentration obtained from detailed equilibrium computations. $\left.\left[V_{2}\right]^{v}\right|^{e q}$ is computed from the knowledge of $V_{2}$ mass fraction at equilibrium condition.

In "real" mechanisms, mass conservation implies that all species mass sums to one. It is not the case for virtual chemistry, where mass conservation is satisfied separately, by optimizing the mixture averaged molecular weight $[40,1]$, regardless of the sum of the mass fractions of species. Constraints are however needed in practice to regularize the optimization problem. By convention, the formalism retained imposes that each set of species mass fractions which constitutes a virtual (main or sub) mechanism sum to unity:

$$
\sum_{k=1}^{N_{s}^{X}} Y_{k}=1
$$

where $N_{s}^{X}$ is the number of species contained in the virtual mechanism X. Consequently, $Y_{V_{2}}$ is computed from Eq. 15 with $N_{s}^{X}=N_{s}^{N O}$ as: 


$$
\left.Y_{V_{2}}\right|^{e q}=1-\left.Y_{F}\right|^{e q}-\left.Y_{O x}\right|^{e q}-\left.Y_{N O}\right|^{e q}-\left.Y_{D}\right|^{e q}-\left.Y_{V_{1}}\right|^{e q}-\left.Y_{V_{3}}\right|^{e q}
$$

$\left.Y_{V_{1}}\right|^{e q}$ and $\left.Y_{V_{3}}\right|^{e q}$ are theoretically equal to zero at equilibrium since the species $V_{1}$ and $V_{3}$ are completely consumed through the reactions $R_{2}^{N O}, R_{4}^{N O}$ and $R_{5}^{N O} .\left.Y_{F}\right|^{e q}$, $\left.Y_{O x}\right|^{e q}$ and $\left.Y_{D}\right|^{e q}$ are known quantities from the main virtual mechanism solution while $\left.Y_{N O}\right|^{e q}$ is assumed equal to the reference one. However, it has been noticed that for very lean and rich conditions $Y_{V_{1}} \neq 0$ at equilibrium. In the 2ZONE optimization, detailed in section 3.3, $Y_{V_{1}}{ }^{e q}$ will be a known quantity at the end of the flame-front block optimization and it is easily accounted for in Eq. 16 for computing $\left.Y_{V_{2}}\right|^{e q}$, before performing the post-flame block optimization.

The reaction orders $F_{V_{1}}^{N O, 2}, F_{N O}^{N O, 3}, F_{V_{3}}^{N O, 4}, F_{V_{3}}^{N O, 5}, F_{N O}^{N O, 6}$ and $F_{V_{2}}^{N O, 6}$ involved in forward reactions $R_{1}^{N O}$ to $R_{6}^{N O}$ are included in the set of optimized parameters. Under equilibrium conditions, the net reaction rate $q_{6}^{N O}$ equals zero. To satisfy this constraint, reaction orders $B_{N O}^{N O, 6}$ and $B_{V_{2}}^{N O, 6}$ of backward reaction $R_{6}^{N O}$ involved in Eq. 13 but must satisfy the following relations [1] :

$$
\begin{aligned}
& B_{N O}^{N O, 6}=F_{N O}^{N O, 6}+1 \\
& B_{V_{2}}^{N O, 6}=F_{V_{2}}^{N O, 6}-1
\end{aligned}
$$

The set of kinetic rate parameters to optimize includes the pre-exponential constants $A_{i}$ the activation energies $E_{a}^{i}$, forward reaction orders $F_{k}^{i}$ and species stoichiometric coefficients $\alpha_{k}^{i}$ for reactions $R_{1}^{N O}$ to $R_{6}^{N O}$ and the temperature exponent $\beta_{T}^{N O, 2}$.

To limit the number of transported species, the species $\mathrm{F}$ and $\mathrm{Ox}$ are identical in both main and NO virtual mechanisms. Consequently, kinetic parameters of reactions $R_{1}^{T}$ and $R_{1}^{N O}$ are identical: $\alpha_{F}^{N O, 1}=\alpha_{F}^{T, 1} ; \alpha_{O x}^{N O, 1}=\alpha_{F}^{T, 1} ; A_{1}^{N O}=A_{1}^{T} ; E_{a}^{N O, 1}=$ $E_{a}^{T, 1} ; F_{F, 1}^{N O}=F_{T, 1}^{N O}$ and $F_{O x, 1}^{N O}=F_{T, 1}^{O x}$.

To capture the sensitivity of NO reaction rate to the equivalence ratio, the optimized pre-exponential constants $A_{2}, A_{4}, A_{5}$ and $A_{6}$ are tabulated as a function of the mixture fraction $Z$ with first-order interpolation.

Reactions $R_{4}^{N O}$ and $R_{5}^{N O}$ are needed to retrieve the slow NO formation and its successive recombination characterizing rich flame conditions, as discussed in section 1. This phenomenon is observed in the NO detailed profiles starting from $\phi=1.4$. In practice, reactions $R_{4}^{N O}$ and $R_{5}^{N O}$ are activated for $\phi \geq 1.4$ through the stoichiometric coefficient $\alpha_{V_{3}}^{N O, 1}$. Section 4.2 discusses the importance of adding reactions $R_{4}^{N O}$ and $R_{5}^{N O}$ for rich conditions. The following section 3 gives details about the optimization procedure.

\section{Optimization of the NO virtual scheme}

\subsection{Principle}

Figure 4 summarizes the general NO mechanism optimization procedure. The vector $\chi^{v}$ contains the ensemble of selected kinetic rate parameters for the virtual NO submechanism, as discussed in the previous section. $N$ random individuals, corresponding to $N \chi_{n}^{v}$ vectors, evolve for a certain number of generations $N_{g e n}$, in the optimization loop, according to the evolutionary algorithm designed by Cailler et al. [40]. Each 


$$
N_{\text {gen }} \text {, the best individual (best } \chi^{v} \text { vector) is identified as output. }
$$



Figure 4.: Schematic view of the general procedure employed to optimize the kinetic rate parameters of the $\mathrm{NO}$ virtual mechanism.

parameter of the vector $\chi_{n}^{v}$ may mutate in the genetic optimization loop within userdefined lower and upper bounds. After a pre-defined number of genetic generations $N_{\text {gen }}$, the best individual (best $\chi^{v}$ vector) is identified as output.

As discussed in [1], to capture pollutant formation in multi-mode combustion, the learning database must include information from both 1-D premixed flames and 1-D non-premixed counterflow flames. To account for these two different flamelet archetypes, the cost function $C_{N O}$ used in the optimization procedure is computed as:

$$
C_{N O}=C_{N O}^{P}+C_{N O}^{N P}
$$

where $C_{N O}^{P}$ and $C_{N O}^{N P}$ are the cost functions relative to premixed flamelet library and non-premixed one, respectively. $C_{N O}^{P}$ is defined from the NO mass fraction as follows:

$$
C_{N O}^{P}=\sum_{i=1}^{N_{\phi}} \frac{\left\|Y_{N O}^{P, v}\left(\phi_{i}^{0}\right)-Y_{N O}^{P, d}\left(\phi_{i}^{0}\right)\right\|_{L_{2}}}{\left\|Y_{N O}^{P, d}\left(\phi_{i}^{0}\right)\right\|_{L_{2}}}
$$

where $Y_{N O}^{P, v}$ and $Y_{N O}^{P, d}$ are the NO mass fraction of a freely propagating premixed flame computed with the virtual and detailed mechanism, respectively. The introduction of the L2 norm $\|\varphi\|=\sqrt{\int_{-\infty}^{+\infty} \varphi(x)^{2} d x}$ provides a global flame criteria and avoid local issues where $\mathrm{Y}_{N O}$ approaches zero. $N_{\phi}$ is the number of premixed flamelets included in the learning database. $\phi_{i}^{0}$ is the fresh gases equivalence ratio of the $\mathrm{i}^{\text {th }}$ flamelet. $x$ is the premixed flame coordinate normal to the flame front. $x \in \mathcal{A}^{P}$, where $\mathcal{A}^{P}$ is the spatial subspace targeted during the optimization procedure for each premixed flamelet. If the whole flame domain is targeted during the optimization process, then $\mathcal{A}^{P}=[-\infty,+\infty]$, where $x=-\infty$ and $x=+\infty$ correspond to fresh and burn gases conditions, respectively.

$C_{N O}^{N P}$ is defined from the NO mass fraction maximum value along the non-premixed 
flame domain:

$$
C_{N O}^{N P}=\sum_{j=1}^{N_{a}} \frac{\max _{y}\left|Y_{N O}^{N P, v}\left(y, a_{j}\right)\right|-\max _{y}\left|Y_{N O}^{N P, d}\left(y, a_{j}\right)\right|}{\max _{y}\left|Y_{N O}^{N P, v}\left(y, a_{j}\right)\right|}
$$

where $Y_{N O}^{N P, v}$ and $Y_{N O}^{N P, d}$ are the NO mass fraction of a non-premixed counterflow flame computed with the virtual and detailed mechanism, respectively. $N_{a}$ is the number of non-premixed flamelets included in the learning database and $a_{j}$ is the strain rate of the $\mathrm{j}^{\text {th }}$ flamelet. $a_{j} \in \mathcal{A}^{N P}$, where $\mathcal{A}^{N P}=\left\{a_{0}, a_{N_{a}}\right\}$ is the range of strain rates targeted. If the whole ensemble of steady state non-premixed flamelets is considered, then $a_{0}=0 s^{-1}$ and $a_{N_{a}}=a_{q}$ where $a_{q}$ is the strain rate at quenching. $y$ is the 1-D flame coordinate. Two optimization strategies, based on 1 or 2 zones, are now compared in the following sections.

\subsection{Single zone optimization (1ZONE)}

The 1-zone optimization (1ZONE) is a brute-force approach that consists in optimizing all reactions rate parameters included in the vector $\chi^{v}$ in one step. During this step, the whole spatial dimension of the $N_{\phi}$ reference premixed flames is targeted: $\mathcal{A}^{P} \in$ $[-\infty,+\infty]$. Simultaneously the entire range of steady state strained non-premixed flamelets is also considered: $\mathcal{A}^{N P} \in\left[a_{0}, a_{q}\right]$. During this step, all kinetic parameters of reactions $R_{1}^{N O}-R_{6}^{N O}$ are optimized. As it will be shown further, the too high number of kinetic rate parameters optimized in a single step causes the failure of the optimization algorithm. A 2-zone optimization method is therefore introduced.

\subsection{2-zone optimization (2ZONE)}

The optimization procedure is split into two consecutive steps dedicated to the optimization of fast and slow NO formations process, respectively. To reduce the number of free kinetic rate parameters to account at each step, reaction $R_{1}^{N O}-R_{6}^{N O}$ are optimized through the two following steps:

(1) Flame front block optimization. Virtual elementary reactions $R_{1}^{N O}-R_{3}^{N O}$ designed to capture flame front $\mathrm{NO}$ formation are trained to reproduce only fast time scales phenomena. Fast time scales learning regions representative of NO formation need to be extracted from the whole set of target flames, which gathers here premixed and non-premixed flames.

(2) Post-flame block optimization. The virtual elementary reactions $R_{4}^{N O}-R_{6}^{N O}$ are optimized to recover the post-flame NO formation which mostly characterize premixed flames burnt gases. During the post-flame block optimization, kinetic parameters of reactions $R_{1}^{N O}-R_{3}^{N O}$, issued from the first step, are conserved. To ensure consistency between all reactions, the cost function is defined on the whole spatial space covered by targeted flamelets, including both flame-front and post-flame regions.

A criterion is required to distinguish the learning subspaces dedicated to the Flame front and Post-flame optimization steps, respectively. The definition of the criterion and the subspaces separation procedure is detailed in Appendix A. For that 
purpose, two physical quantities $\left(\delta_{F F}\right.$ and $\left.a_{F F}\right)$, dedicated respectively to premixed and non-premixed flamelets, are defined to distinguish the fast NO formation time scale from the slow one. $\delta_{F F}$ is the critical length scale for premixed flamelets. For $x \in\left[-\infty, \delta_{F F}\right]$, fast time scales, characteristics of prompt NO, dominate. At the opposite thermal and reburning are more important for $x \in\left[\delta_{F F},+\infty\right] . a_{F F}$ is the critical strain rate for non-premixed strained flamelets. For $a \in\left[a_{F F}, a_{q}\right]$ fast NO time scales dominate while for $a \in\left[0, a_{F F}\right]$ slow time scales are more important.

The optimization process is then split into two steps:

Step 1 The Flame front block is optimized using flame data characteristic of prompt NO process. In practice, the cost functions $C_{N O}^{P}$ and $C_{N O}^{N P}$, computed by Eqs. 20 and 21 , are restricted to the subspaces $\mathcal{A}^{P} \in\left[-\infty, \delta_{F F}\right]$ and $\mathcal{A}^{N P} \in\left[a_{F F}, a_{q}\right]$. During this step, only kinetic parameters of reactions $R_{1}^{N O} R_{3}^{N O}$ are optimized.

Step 2 The Post-flame block is optimized using flame data characteristic of slow NO processes. In practice, the cost functions $C_{N O}^{P}$ and $C_{N O}^{N P}$, computed by Eqs. 20 and 21 , include the entire premixed flamelet space $\mathcal{A}^{P} \in[-\infty,+\infty]$. Analysis of DNS of partial-oxydation processes have shown that, in post-flame regions characterized by slow chemistry phenomena, molecular diffusion is less important than chemical reaction [43]. According to ILDM theory [28], chemical trajectories followed by premixed and non premixed flames in the composition space are therefore identical. In the present work, only premixed flamelets are therefore targeted to optimize slow NO chemistry: $\mathcal{A}^{N P}=\varnothing$. During this step, only kinetic parameters of reactions $R_{4}^{N O}-R_{6}^{N O}$ are optimized.

The two learning procedures, 1ZONE and 2ZONE, are now compared on a simple test case.

\subsection{Comparison of $1 Z O N E$ and 2ZONE optimization procedures}

The learning database retained to optimize the NO virtual mechanism is made of a single stoichiometric $\mathrm{CH}_{4}$ /air premixed flamelet. Both 1ZONE and 2ZONE optimized solutions are a-posteriori compared against the targeted solution in Fig. 5. The slow NO formation, visible over the entire computational domain, from $x=0$ to $x=5 \mathrm{~m}$, is fairly well captured by the two algorithms. But the 1ZONE (dashed line) optimized scheme fails to capture the prompt NO formation, as evidenced in the inner graph which focuses on the thermal flame thickness (from $x=0$ to $x=25 \mathrm{~mm}$ ). This inaccuracy is corrected by the 2ZONE (dotted line) algorithm which is able to predict both fast and slow NO chemistry.

To understand the difference between 1ZONE and 2ZONE, the error $\epsilon$ between the targeted flame data and the two optimized solutions a-posteriori obtained with the virtual mechanism is introduced:

$$
\epsilon\left(\mathcal{A}^{\prime}\right)=\frac{\left\|Y_{N O}^{P, v}\left(x, \phi^{0}\right)-Y_{N O}^{P, d}\left(x, \phi^{0}\right)\right\|_{L_{2}}}{\left\|Y_{N O}^{P, d}\left(x, \phi^{0}\right)\right\|_{L_{2}}} \quad \text { for } \quad x \in \mathcal{A}^{\prime}
$$

where $\mathcal{A}^{\prime}$ is the 1-D spatial subspace on which the error $\epsilon$ is computed. The three subspaces $\mathcal{A}^{\prime}=[-\infty,+\infty], \mathcal{A}^{\prime}=\left[-\infty, \delta_{F F}\right]$ and $\mathcal{A}^{\prime}=\left[\delta_{F F},+\infty\right]$ are retained to measure $\epsilon$ along the the whole flame, flame front region and post-flame region, respectively. Figure 6 plots the evolution of these three errors as a function of the generation num- 


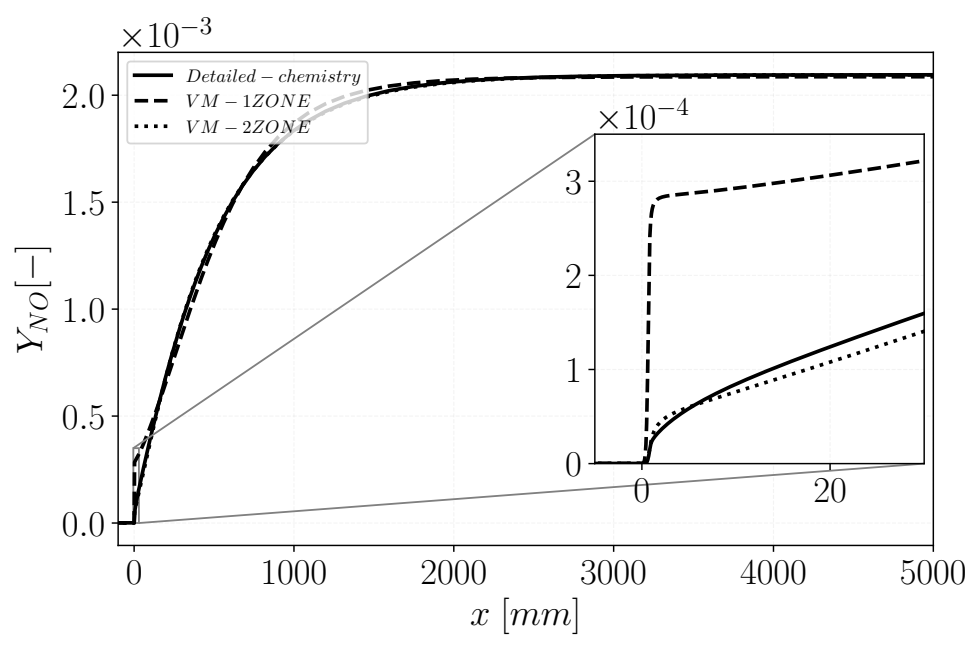

Figure 5.: Comparison of the NO mass fraction computed with virtual chemistry and reference detailed chemistry, at stoichiometric conditions. Virtual chemistry results are shown for the 1-zone optimization (1ZONE) and the 2-zone optimization (2ZONE). The comparison is proposed either over the whole computational domain and in the flame front region.

ber $N_{\text {gen }}$ for both 1ZONE and 2ZONE algorithms.
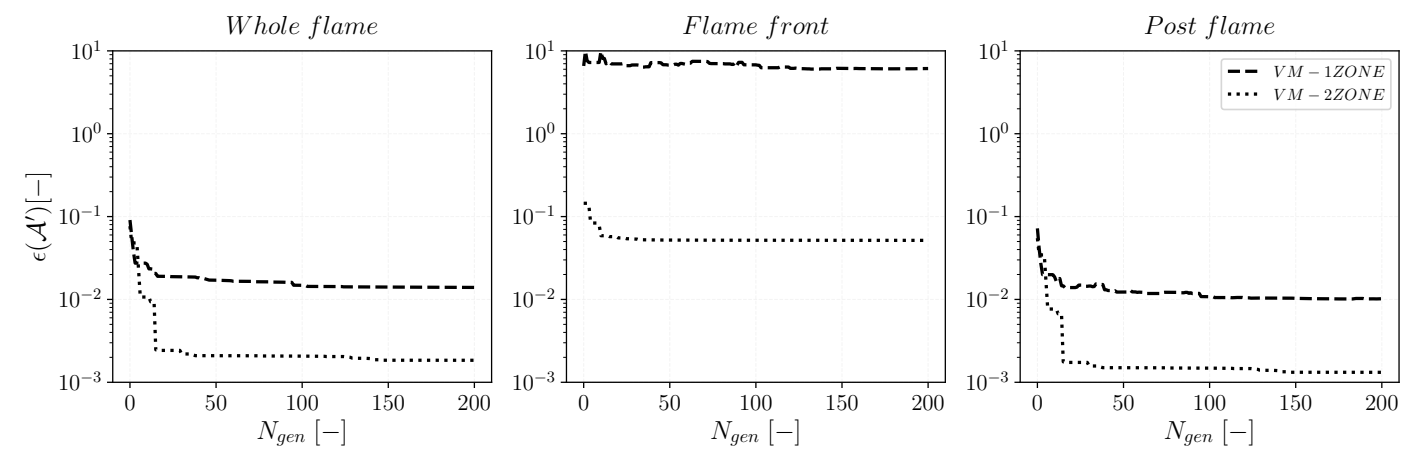

Figure 6.: Error evolution during the optimization procedure. $N_{g e n}$ is the generation number performed by the evolutionary algorithm. The error is shown for the single zone optimization (VM-1ZONE) and for the two zone optimization (VM-2ZONE) and it is computed using three subspaces: whole flame, flame front and post-flame regions.

The error computed over the whole domain decreases as expected with the 1ZONE procedure during 15-20 generations and then it reaches a plateau around 2\% (Fig. 6 , left). However, better results are observed with the 2ZONE mechanism. With the 1ZONE procedure, a very poor convergence of the error is observed in the flame front, which stays above $1000 \%$ (Fig. 6 , center). The failure of the optimization process is supposed to be due to:

- the high number of kinetic rate parameters optimized in a single step,

- the wide range of values covered by these parameters to capture both fast and slow NO chemistry pathways. 
Figure 7 describes the 2ZONE optimization procedure.

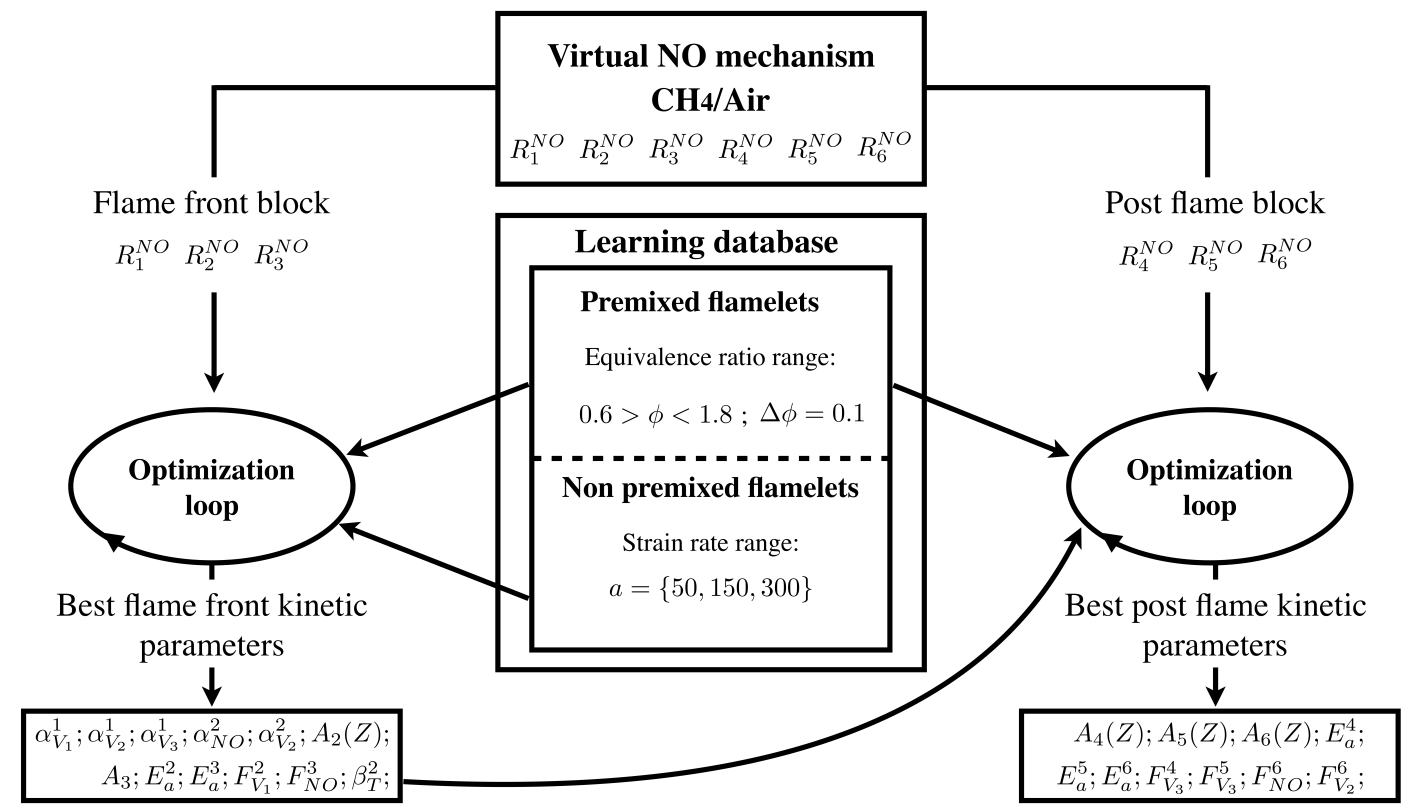

Results are greatly improved by the 2ZONE algorithm where the convergence of the error in the flame front is ensured. As a consequence, the error on the prompt NO prediction decreases below $5 \%$ after 20 generations. In addition, the post-flame error of the 2ZONE optimization procedure is also decreased by more than an order of magnitude compared with the 1ZONE optimization procedure (Fig. 6, right).

\section{Results: 1-D flames}

This section presents the 1-D results of the $R_{1}^{N O}-R_{6}^{N O}$ NO virtual mechanism optimized according to the 2ZONE strategy over $\mathrm{CH}_{4}$ /air hybrid flamelets. The mechanism is reported in Appendix. B. Results for premixed freely propagating flames, for several mixture equivalence ratios, and for diffusion flames for several flame strain rates are presented and compared to simulations carried out with the detailed kinetic mechanism.

\subsection{Virtual mechanism optimization for multi-mode combustion}

Figure 7.: Virtual NO mechanism optimization procedure applied to $\mathrm{CH}_{4}$ /air combustion. The 2ZONE optimization is applied.

The reference detailed chemistry premixed and non-premixed flamelets are computed with the REGATH solver [5] by accounting for differential diffusion and by using the GRI3.0 mechanism [3]. The $N_{\phi}$ premixed flamelets retained to compute the cost function $C_{N O}^{P}$ from Eq. 20 cover the whole flammability limit: $0.6<\phi_{0}<1.8$. Varying the number of targeted flames $N_{\phi}$, we found an empirical optimal compromise retaining $N_{\phi}=13$ (or equivalently $\Delta \phi=0.1$ ). For 1 -D non-premixed counterflow flames, NO profiles representative of flames having a strain rate $a$ greater than the critical value $a_{F F}=50 \mathrm{~s}^{-1}$ are included. As for premixed flames, an empirical optimal solution was 
found for the strain-rate discretization $\Delta a$ so as to ensure a compromise between the convergence of the optimization algorithm and the precision of the mechanism over the whole range of strain rates. For this application, the subspace $\mathcal{A}^{N P}=\{50,150,300\}$ $\mathrm{s}^{-1}$ is retained to build up the non-premixed learning library.

Three virtual mechanisms of different sizes are generated in order to assess the influence of the number of virtual species / reactions. The complete virtual mechanism $R_{1}^{N O}$ $R_{6}^{N O}$ is indicated with the abbreviation VM-6R. Two smaller mechanisms VM-4R and VM-5R are also considered. VM-4R is obtained by removing $R_{4}^{N O}$ and $R_{5}^{N O}$ from the VM-6R mechanism. Whereas three reactions $R_{1}^{N O}-R_{3}^{N O}$ are dedicated to capture fast processes, only $R_{6}^{N O}$ is kept to handle slow time scales and to retrieve equilibrium conditions. At the opposite VM-5R is designed by removing $R_{3}^{N O}$ from the VM-6R mechanism. The three elementary reactions $R_{4}^{N O}{ }_{-} R_{6}^{N O}$ are retained for slow process, while the number of "fast" elementary reactions is restricted to $R_{1}^{N O}$ and $R_{2}^{N O}$. Table 1 summarizes the three mechanism's properties.

Table 1.: Summary of the three optimized virtual NO mechanisms. The virtual reactions that are included in each mechanism are indicated.

\begin{tabular}{ccccc}
\hline Mechanisms & Reactions & $\begin{array}{c}\text { Reaction nb. for } \\
\text { Flame front }\end{array}$ & $\begin{array}{c}\text { Reactions nb. for } \\
\text { Post flame }\end{array}$ & Species nb. \\
\hline \hline VM-6R & $R_{1}^{N O}-R_{6}^{N O}$ & 3 & 3 & 6 \\
\hline VM-4R & $\begin{array}{c}R_{1}^{N O}-R_{3}^{N O} \\
R_{6}^{N O}\end{array}$ & 3 & 1 & 5 \\
\hline VM-5R & $\begin{array}{l}R_{1}^{N O}-R_{2}^{N O} \\
R_{4}^{N O}-R_{6}^{N O}\end{array}$ & 2 & 3 & 6 \\
\hline
\end{tabular}

Molecular diffusive fluxes of species belonging to both main and NO virtual schemes are modeled with a unity Lewis number assumption. However, as discussed in [1], this assumption still enable a correct prediction of unstretched laminar flame consumption speed and species profiles across planar flame fronts, as the targeted 1-D flame solutions used to calibrate the main virtual scheme include differential diffusion effects.

\subsection{Premixed flames}

VM-6R results are a-posteriori compared versus the reference detailed chemistry solutions, for three different equivalence ratios: lean $(\phi=0.6)$, stoichiometric $(\phi=1.0)$ and rich $(\phi=1.6)$. The results are presented in the flame front and in the post-flame regions in Fig.8. The proposed 6-reaction mechanism allows to correctly reproduce prompt and thermal post-flame NO formation characterizing lean and stoichiometric conditions. The post-flame NO re-burning phenomena, encountered in rich flame conditions, are also correctly described.

A comparison is now proposed to stress the role of reactions $R_{4}^{N O}$ and $R_{5}^{N O}$ in the mechanism. Figure 9 compares, for a rich $(\phi=1.6)$ and a stoichiometric flames, the results obtained with the two mechanisms VM-4R and VM-6R against the detailed chemistry solutions. It shows that the two reactions $R_{4}^{N O}$ and $R_{5}^{N O}$ are required to capture the slow NO reburning already illustrated in Fig. 1. These reactions are however not necessary for lean, stoichiometric and moderately rich conditions $(\phi<1.4)$, where reburning phenomena is not significant. Consequently VM-4R and VM-6R solutions 

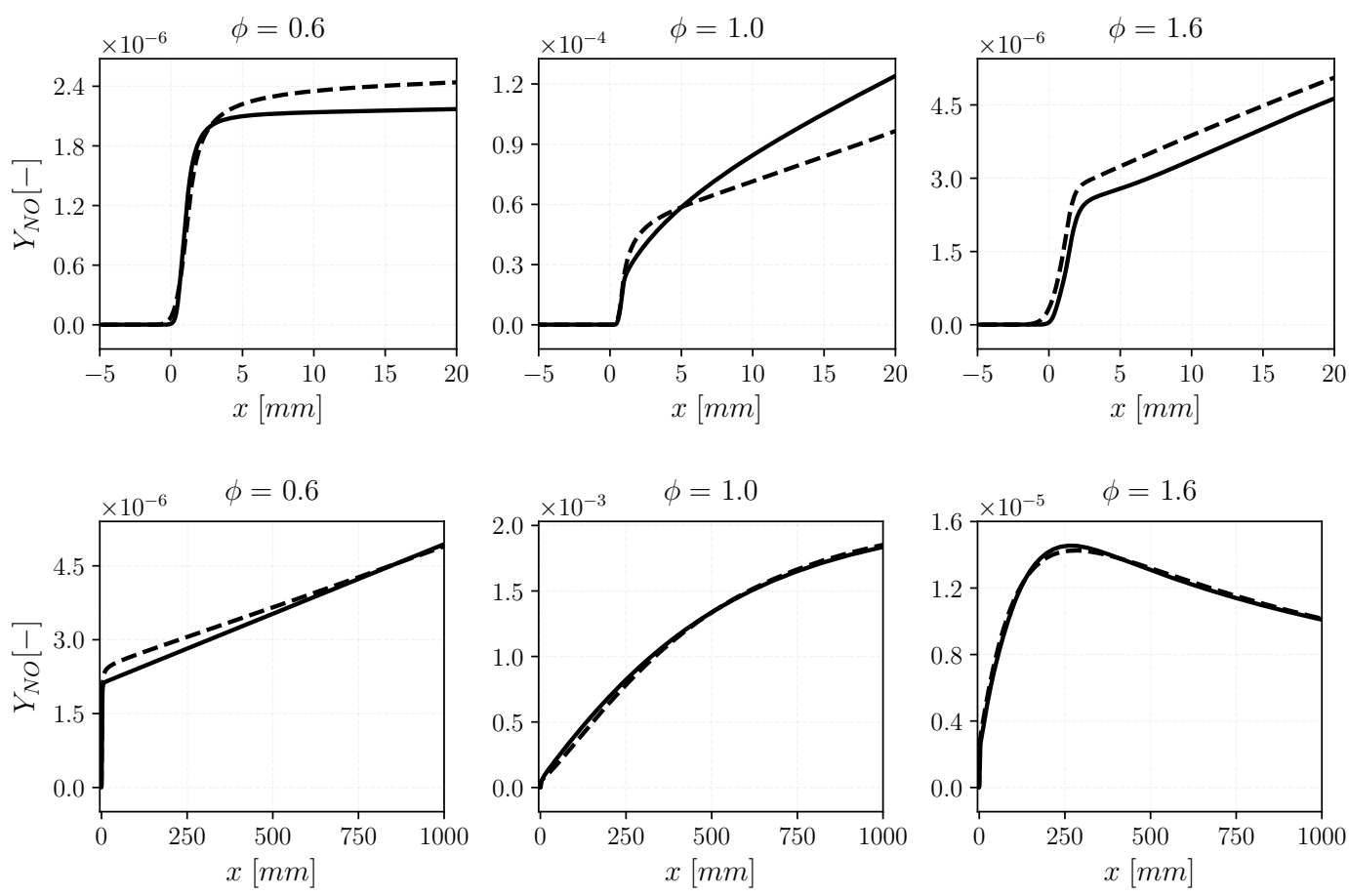

Figure 8.: NO mass fraction profiles for a lean $(\phi=0.6)$, a stoichiometric $(\phi=1.0)$ and a rich $(\phi=1.6)$ equivalence ratio. Virtual chemistry VM-6R NO (dashed lines) is compared versus the reference detailed chemistry (solid lines). Results are presented at the flame front (upper figures) and post-flame (lower figures) spacial scales.

collapse on the same curve for $\phi<1.4$ as illustrated in Fig. 9 (right).

\subsection{Non-premixed flames}

1-D non-premixed counterflow flames are a-posteriori computed with the entire optimized virtual mechanism VM-6R. Figure 10 shows the NO profile computed with the whole virtual NO mechanism VM-6R, and the reference profiles, for two different flame strain rates. The proposed virtual NO mechanism optimized to fit both premixed and non-premixed flame archetypes retrieves the high sensitivity of the NO profile to the flame strain rate. Decreasing the flame strain rate, the residence time increases and consequently the NO formation is higher. However, virtual NO mechanism VM-6R does not perfectly describe the fast NO mass fraction decay on the flame rich side, which characterizes detailed chemistry profiles. It turns out difficult to find a better compromise between premixed and non-premixed flames in the flame front region with only two reactions $\left(R_{2}^{N O}-R_{3}^{N O}\right)$.

Comparison between VM-6R and VM-5R, in which reaction $R_{3}^{N O}$ has been removed before the optimization process, is also shown in Fig. 10. The large discrepancy observed between VM-5R and the detailed chemistry solutions confirms that $R_{3}^{N O}$ is required in the flame front block to capture NO formation in non-premixed flames. Without it, the virtual mechanism does not have enough degrees of freedom to capture NO formation in both premixed and non-premixed flame regimes. 

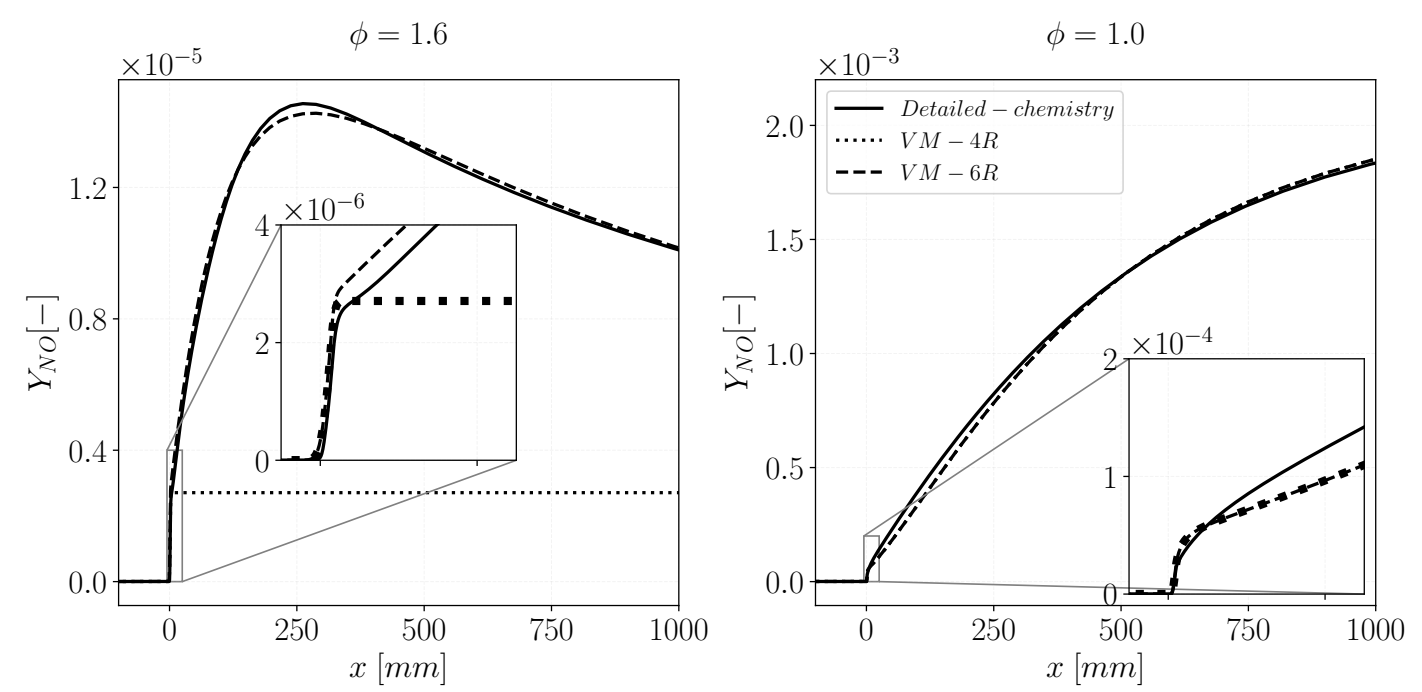

Figure 9.: Comparison of NO mass fraction from detailed chemistry and virtual chemistry for a rich (left) and a stoichiometric (right) 1-D premixed flames. Two virtual mechanism are shown: the first retains 1 reaction only in the post-flame block (VM-4R) and the second retains the whole post-flame block (VM-6R).



Figure 10.: NO mass fraction profiles in non-premixed counterflow flames for two strain rate values (150 and $300 \mathrm{~s}^{-1}$ ). Detailed chemistry and virtual chemistry results are compared. Two virtual mechanisms are shown: the first considers 2 reactions only in the flame front block (VM-5R) and the second considers the whole flame front block (VM-6R). Different colors are used for different stain rates.

\section{2-D slots burner flames}

In the current section, virtual chemistry is challenged in 2-D CFD computations and compared against detailed chemistry simulations. The simulations are carried out for several 2-D laminar flame benchmarks including a premixed, a non-premixed and a partially premixed flame. 
The CFD code laminarSMOKE [42] is used to perform the computations for both detailed chemistry and virtual chemistry. The laminarSMOKE code is based on the open-source suite OpenFOAM [44]. It has already shown capability to accurately model laminar flames including detailed chemistry using hundreds of species and reactions $[45,46]$. The transport equations of mass, momentum, energy, and species are solved based on the operator-splitting approach [47].

\subsection{Premixed flame}

The premixed single slot burner geometry consists of a 2-D rectangular computational domain whose dimensions are shown in Fig. 11. The boundary conditions include an inlet, an outlet and adiabatic walls. Since the configuration is axial-symmetric only a half of the computational domain is simulated. The other half of the computational domain is obtained by symmetric reflection of the first part. Therefore a symmetry boundary condition is imposed along the axis.
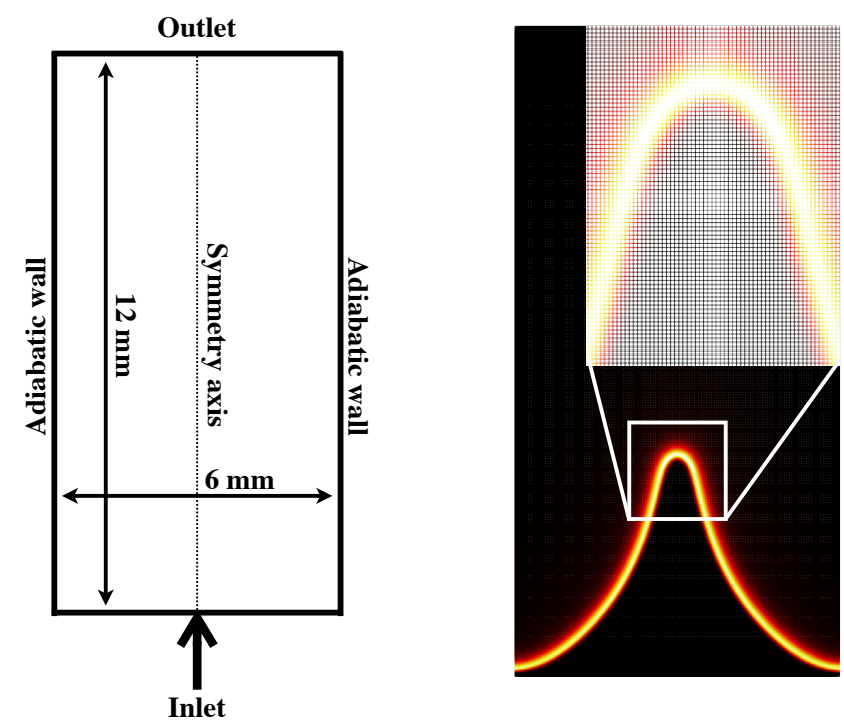

Figure 11.: 2-D premixed slot burner numerical set-up. On the left schematic view of the computational domain with the corresponding dimensions and the boundary conditions. On the right the normalized heat release rate from numerical simulation shown on the computational grid.

At the inlet, a developed laminar parabolic velocity profile is prescribed in the axial direction using a mean value $U_{m}=0.6 \mathrm{~m} / \mathrm{s}$, whereas the others velocity components are set equal to zero. A cartesian grid is considered in the simulation. The characteristic cell size is of about $\sim 0.015 \mathrm{~mm}$. This cell size allows to fully resolve the thermal flame thickness and the heat release rate of a premixed laminar flame. In particular, the employed mesh size ensures 15 to 20 cells across the thermal flame thickness. A zoom on the computational grid, in the flame tip region, is made in Fig. 11 and an example of normalized heat release rate is also shown on the computational grid. Indeed the heat release rate is properly solved over the employed grid.

Figure 12 shows the temperature and NO field for the 2-D premixed burner at 
three different equivalence ratios $(0.8,1.0$ and 1.2). Virtual chemistry results are directly compared to detailed chemistry ones. The temperature field predicted by the main virtual mechanism is in good agreement with the detailed chemistry, for the three equivalence ratios. It is remarkable that the flame height is properly captured by virtual chemistry. At $\phi=1.2$ a misprediction by about $10 \%$ is however observed, which is attributed to species transport phenomena. As discussed in [1], as long as 1-D detailed chemistry flamelets computed under differential diffusion assumptions are targeted, differential diffusion phenomena in the direction normal to the flame front are well captured, even if unity Lewis number assumption is made to close the virtual species balance equations. In 2-D and 3-D, molecular diffusions effects are however not correctly tackled, causing a difference in the flame curvature description and on the flame height, especially visible under rich conditions.

The virtual NO field agrees well with the temperature field: when temperature increases earlier for virtual chemistry (as for $\phi=1.2$ ), NO increases earlier too. The 2-D slot burner configuration, retained in the present work allows to observe mostly the whole prompt NO formation and the first part of the thermal one. However, as observed in the 1-D premixed profiles in Fig. 9, NO is further produced in the burnt gases, for longer residence time, through the thermal route. An underestimation of the NO level is observed at $\phi=0.8$ and 1.0, whereas a better prediction is observed for $\phi=1.2$. This behavior is in accordance with the 1-D profiles shown in Fig. 8 at the flame front scale.

For the stoichiometric slot burner flame, 1-D temperature and NO mass fraction profiles are extracted from the 2-D field over both axial and a radial directions. The 1-D sections, used for the comparison, are indicated by white lines in Fig. 12. Figure 13 shows the temperature and NO profiles from virtual and from detailed chemistry computations along the two lines in the axial direction $(\mathrm{x}=0 \mathrm{~mm})$ and for a radial section $(y=2 \mathrm{~mm})$. The temperature agreement in the axial direction confirms that the flame height is well retrieved even if a slight shifting in the radial direction is observed. This discrepancy might be due to the non-perfect reproduction of flame curvature effects.

\subsection{Non-premixed flame}

A 2-D non-premixed laminar coflow flame is simulated in the present section. The considered configuration along with the main burner dimensions and the boundary conditions set-up are schematized in Fig. 14. The figure also shows a normalized heat release rate field over half of the computational domain region close to the burner nozzle. A fuel jet is surrounded by a pure air coflow allowing the development of a non-premixed diffusion flame. The computational geometry is symmetric with respect to the centreline axis, as for the premixed single slot burner; consequently, just a half of the computational domain is computed. Adiabatic walls are imposed close to the air inlet whereas an inlet/outlet boundary condition [44] is prescribed on the burner side.

A 2-D non uniform structured rectangular mesh is used in the simulations as proposed by Cuoci et al. [42, 45]. The characteristic mesh size in the flame front region is of about $\sim 0.05 \mathrm{~mm}$ which is sufficient to ensure a proper flame resolution in diffusion flame conditions. Velocity boundary conditions are tuned to ensure the flame attachment to the burner lip avoiding any flame lift-off. Flat velocity profiles 


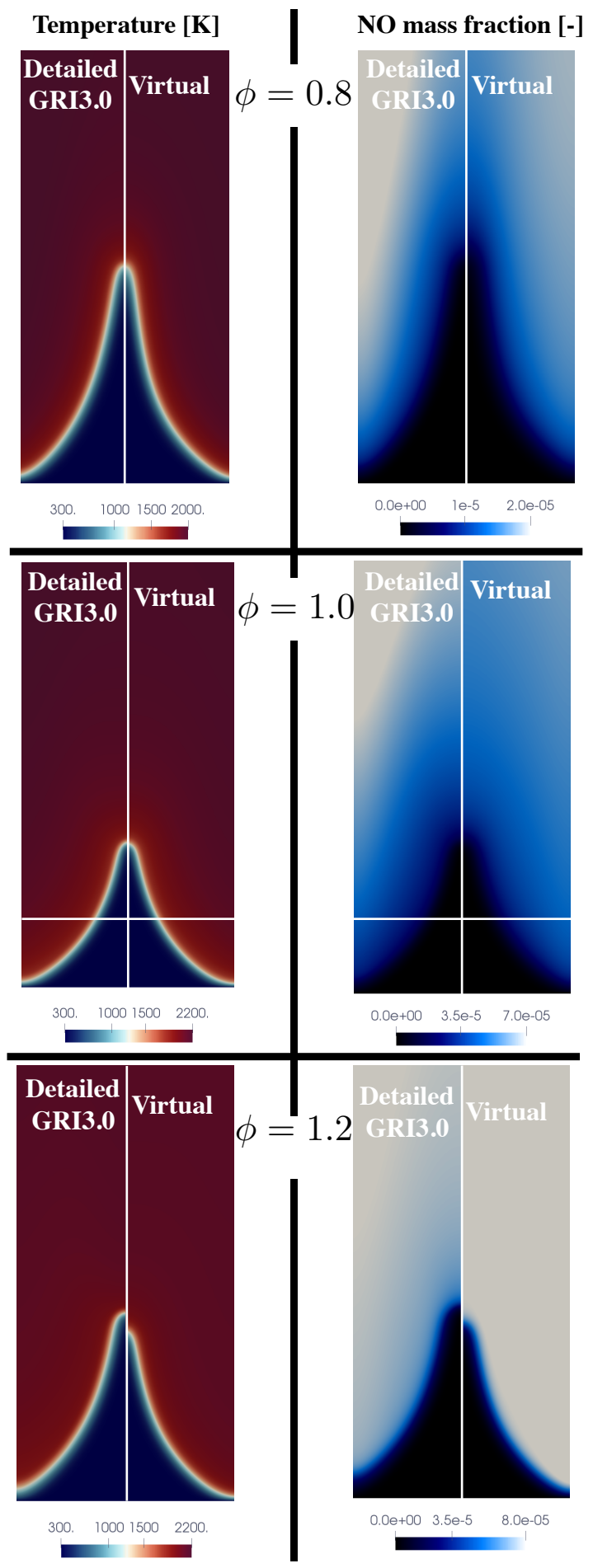

Figure 12.: Temperature and NO mass fraction colormaps for the 2-D laminar single slot burner (Bunsen flame). Virtual chemistry solution is compared with detailed chemistry one. 

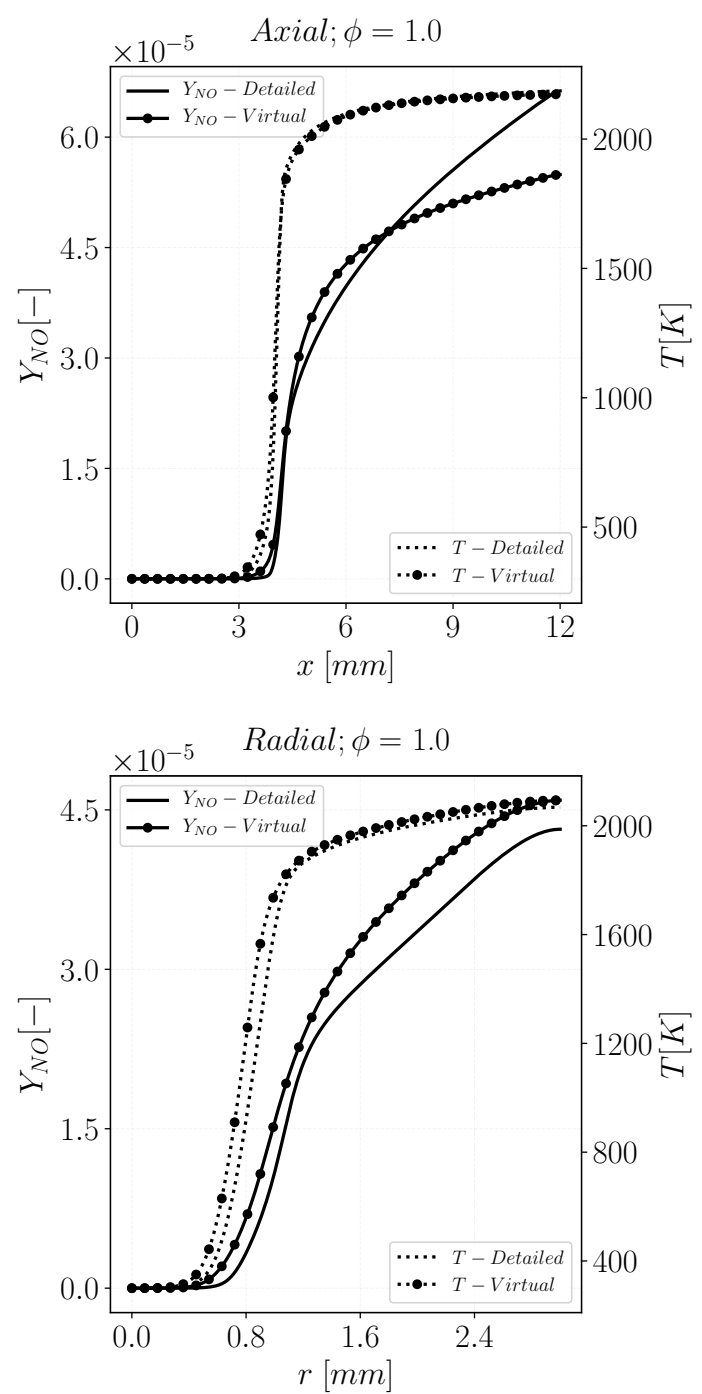

Figure 13.: Virtual chemistry (symbols + lines) is compared against detailed chemistry (lines) for temperature (dashed lines) and NO mass fraction (solid lines). Top: axial profiles. Bottom: radial profiles at the centerline distance $\mathrm{x}=2 \mathrm{~mm}$. 

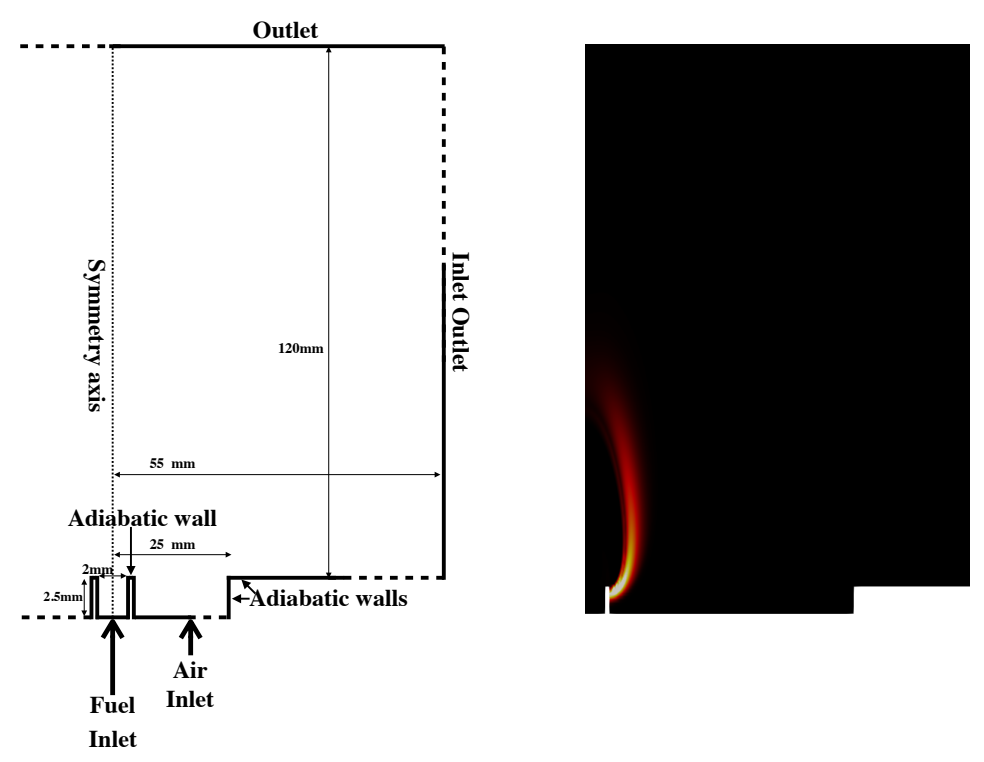

Figure 14.: Numerical set-up of the coflow diffusion flame burner. On the left a schematic view of the 2-D simulated domain is presented. The main burner dimensions and the prescribed boundary conditions type are shown. On the right an example of normalized heat release rate computed from detailed chemistry simulation is shown.

at $0.15 \mathrm{~m} / \mathrm{s}$ are prescribed in the two streams. Pure $C_{4} H_{4}$ and pure air at $300 \mathrm{~K}$ are injected in the Fuel Inlet and the Air Inlet, respectively.

Figure 15 shows the temperature and NO mass fraction fields for the 2-D diffusion flame configuration, comparing virtual chemistry to the detailed chemistry results. The virtual chemistry flame shape is close to the detailed chemistry one, but it appears more compact. In the virtual chemistry simulation, temperature increases faster than with detailed chemistry. The NO mass fraction field predicted by virtual chemistry is correctly located in the computational domain with respect to temperature field.

Figure 16 compares the temperature and NO mass fraction along the flame axis. As virtual chemistry temperature rises faster, NO mass fraction presents an early peak at $x=0 \mathrm{~mm}$. Successively the small NO reburning corresponding to detailed chemistry is overestimated by virtual chemistry. This reburning phenomenon in coflow flame configuration has been already observed by Cuoci et al [42]. The max NO peak prediction, corresponding to the temperature peak is correctly predicted. However, the amplitude of NO mass fraction is relatively overestimated. This phenomenon is attributed to the slight temperature overestimation. After the peak zone, the NO reduction due to consumption and/or dilution effects is correctly captured.

\subsection{Partially-premixed flame}

The developed NO virtual mechanism is finally assessed in a partially premixed flame configuration. The considered geometry and mesh are the same as the diffusion flame configuration, described in the previous section. In the central jet (Fuel Inlet) 

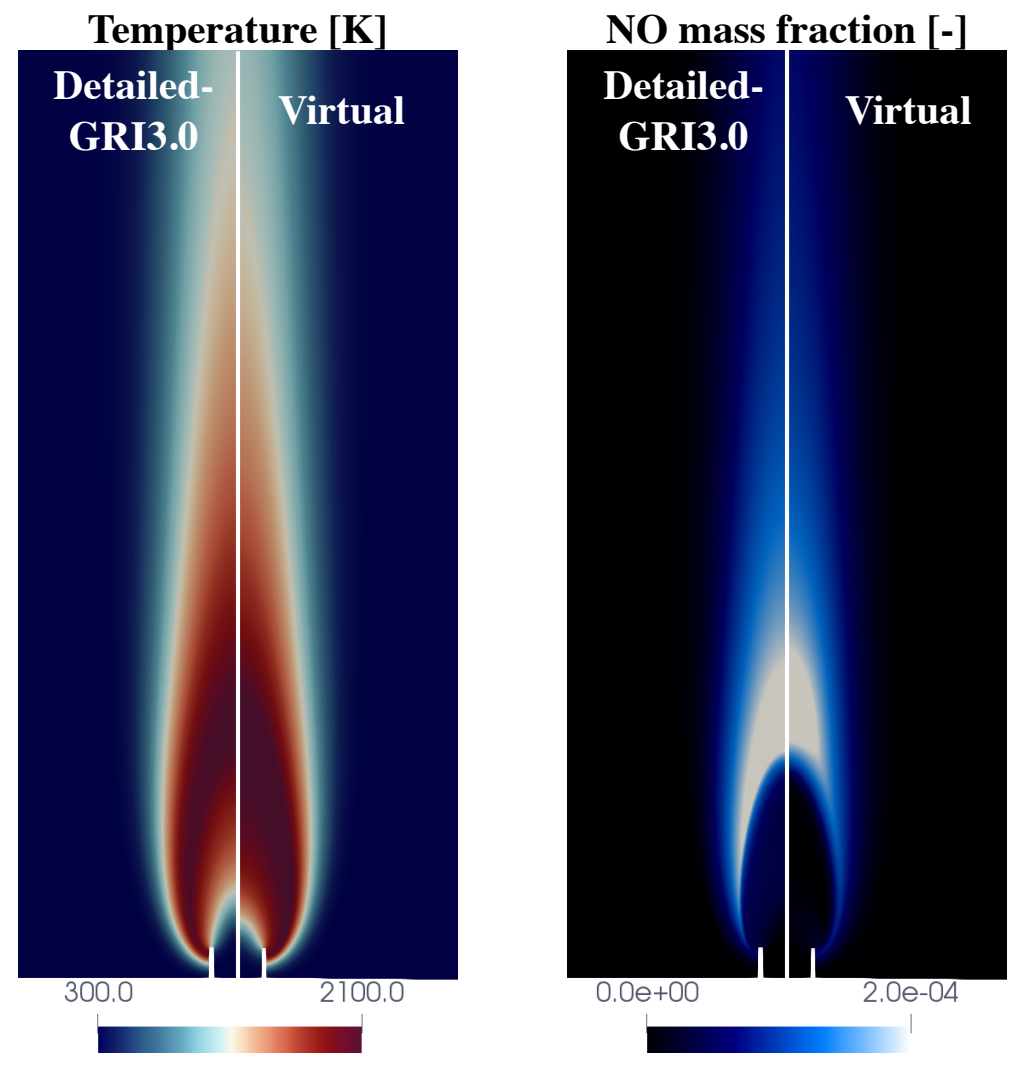

Figure 15.: Temperature and NO mass fraction fields from numerical simulations for the diffusion flame. The virtual chemistry results are compared versus the reference GRI3.0 solutions.

a premixed mixture of fuel and air, above the flammability limit, is injected. An equivalence ratio equal to 2.5 and a temperature of $300 \mathrm{~K}$ are prescribed. Pure ambient air at $300 \mathrm{~K}$, as for the diffusion flame configuration, is injected through the Air Inlet tube. Inlet velocities are tuned to stabilize the flame at the burner lips, without having any lift-off. A flat velocity profiles of $0.35 \mathrm{~m} / \mathrm{s}$ and $0.05 \mathrm{~m} / \mathrm{s}$ are prescribed at the Fuel Inlet and the Air Inlet, respectively.

Figure 17 shows the temperature and NO mass fraction 2-D fields for the partially premixed flame. The flame is stabilized at the same position with detailed and virtual chemistry. NO field is correctly located over the computational domain, but the NO peak value is overestimated.

Figure 18 shows the temperature and NO profiles along the axis. The NO 1-D profile confirms the correct prediction of the NO peak position but its value is overestimated.

\subsection{CPU cost comparison}

A comparison to evaluate the CPU ratio between detailed chemistry and virtual chemistry simulation was carried out. The stoichiometric 2-D premixed slot burner is computed for the same physical time $(10 \mathrm{~ms})$, using the same numerical set-up and em- 


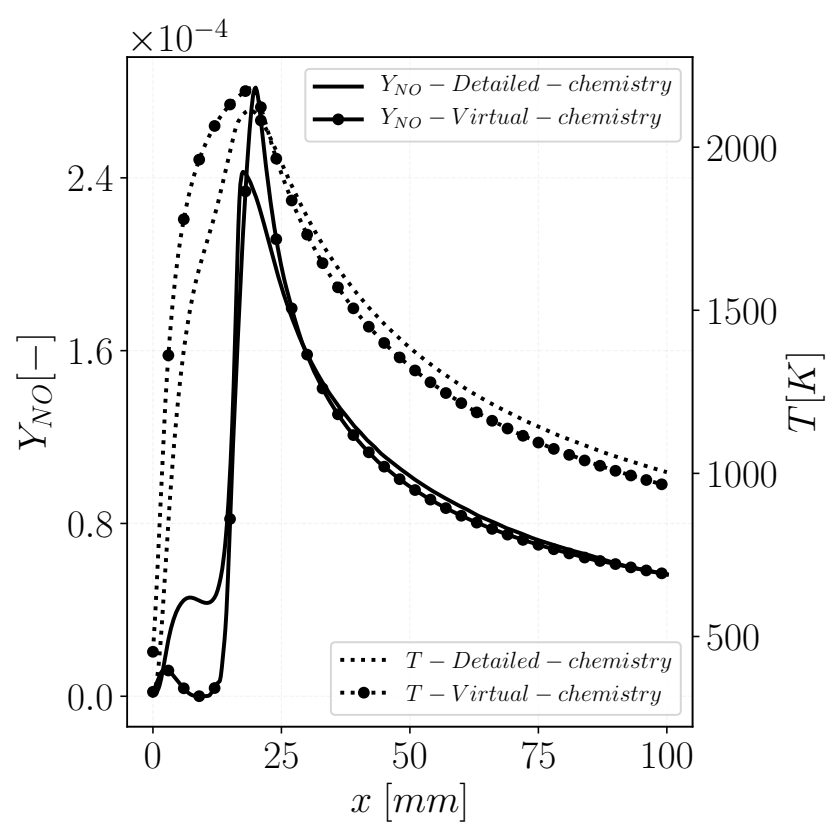

Figure 16.: Temperature (dashed lines) and NO mass fraction (solid lines) along the centerline axis of the 2-D coflow non-premixed flame. Virtual chemistry (symbols + lines) is compared against detailed chemistry (lines).

ploying the same numbers of processors for virtual and detailed chemistry.

Table 2.: CPU cost comparison between detailed reference chemistry and virtual chemistry computations. Main temperature mechanism and the NO one are considered for virtual chemistry.

\begin{tabular}{ccc}
\hline Mechanism & Nb. species & $C=t / t^{v}$ \\
\hline \hline GRI3.0 [3] & 53 & 39 \\
\hline Virtual Mech. & 12 & 1
\end{tabular}

Table 2 summarizes the results of the comparison. The CPU time ratio $C=t^{d} / t^{v}$ that compares the detailed $\left(t^{d}\right)$ and virtual chemistry $\left(t^{v}\right)$ computational time, is equal to $=39$. The drastic CPU cost reduction is mainly due to the species and reactions reduction. The observed CPU speed-up is equal to:

$$
C=\frac{t^{d}}{t^{v}} \simeq\left(\frac{n^{d}}{n^{v}}\right)^{2.5}
$$

where $n^{d}(=53)$ and $n^{v}(=12)$ are respectively the number of species included in the detailed and virtual mechanisms. This result is in accordance with previous numerical studies that use implicit solvers. [48, 49]. This CPU result analysis is valid for any 2-dimensional laminar computation (premixed, non-premixed and partially premixed) performed with laminarSMOKE solver [42]. 

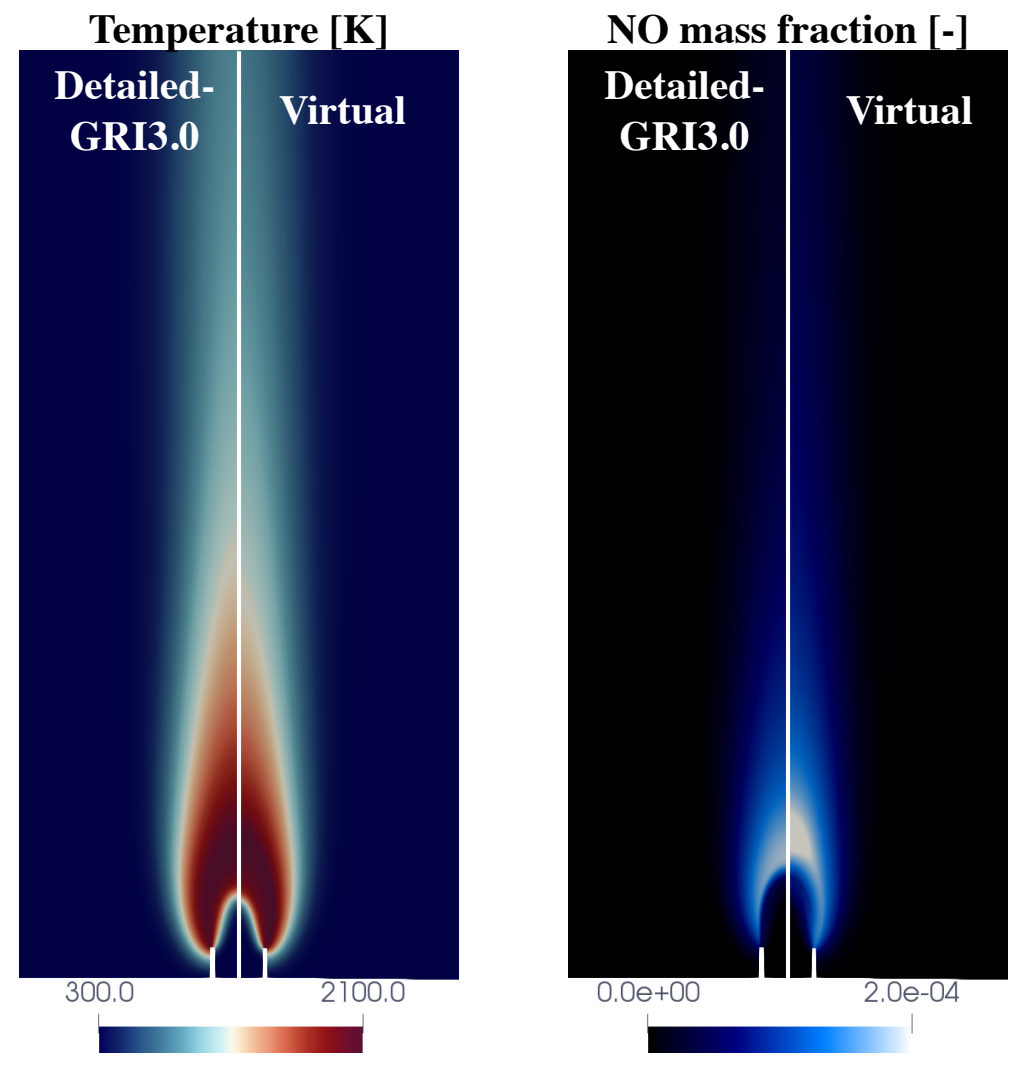

Figure 17.: Temperature and NO mass fraction fields from numerical simulations for the partially-premixed flame. The virtual chemistry results are compared versus the reference GRI3.0 solutions.

\section{Conclusions}

The virtual chemistry strategy has been retained to develop a new pollutant reduced mechanism devoted to NO prediction. The original virtual chemistry formulation has been used to predict flame temperature and heat release rate. An NO virtual mechanism has been designed using a reduced set of virtual species and reactions and trained over a hybrid flamelet database, made of premixed and non-premixed 1-D flames.

A two-step optimization strategy has been developed to separate and to independently optimize the virtual reactions dedicated to flame front and post-flame NO chemistry, respectively. The proposed model is able to describe all the NO chemistry pathways exhibited by the reference flame solution. In particular prompt NO, thermal NO and post-flame NO reburning are correctly described by the virtual chemistry model. The developed NO virtual mechanism has been tested in 2-D laminar premixed, non-premixed and partially premixed flame computations. A comparison to detailed reference chemistry has also been proposed. In the CFD computations, the final CPU cost associated with the virtual chemistry simulation is drastically smaller than with the detailed chemistry one (of about 40 times).

The thermodynamics and kinetics parameter are automatically optimized but the design of the virtual chemistry architecture remains based on a physically-based approach. Despite the promising application to NO chemistry, the empirical identification of the optimal number of virtual species and reactions network may become problem- 


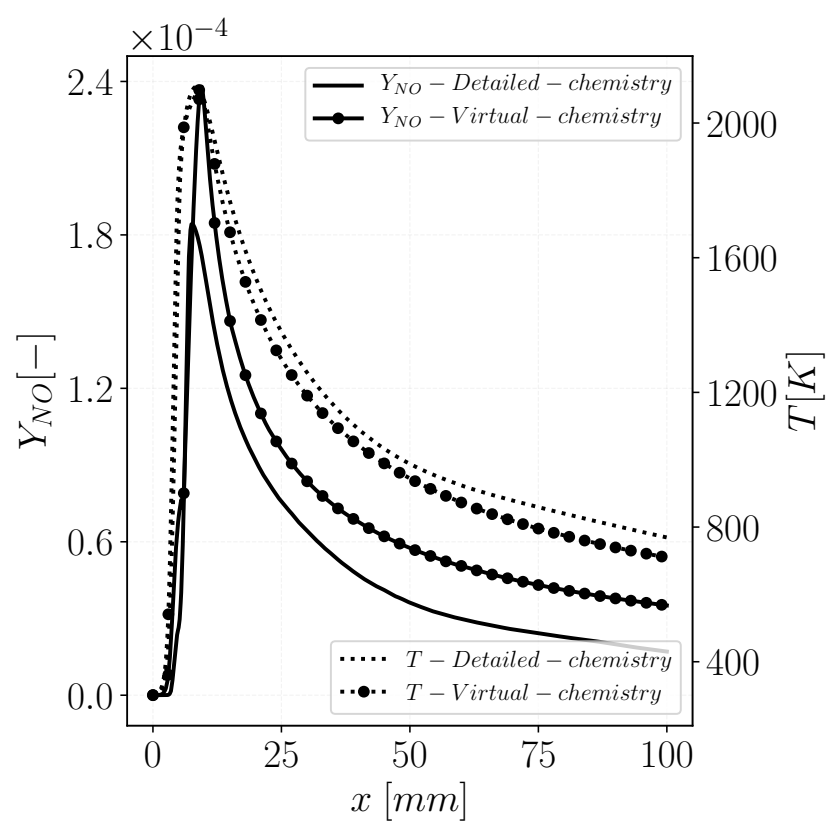

Figure 18.: Temperature (dashed lines) and NO mass fraction (solid lines) along the centerline axis of the 2-D coflow partially premixed flame. Virtual chemistry (symbols + lines) is compared against detailed chemistry (lines).

atic in more complex systems where relevant chemical time scales and trajectories are more numerous. To overcome this current limitation, automatic methods to build-up of the virtual chemistry network, from a time scale analysis of the detailed chemical scheme, are under investigation.

\section{Acknowledgements}

The authors warmly acknowledge Prof. Nasser Darabiha for the useful discussions about the model and the numerical simulations. This project has received funding from the European Union's Horizon 2020 research and innovation programme under the Marie Sklodowska-Curie grant agreement No 643134. This work was granted access to the HPC resources under the allocations $A 0032 B 10253$ made available by GENCI (Grand Equipement National de Calcul Intensif).

\section{Appendix A. NO optimization criterion: zone separation and learning database definition}

The objective is to find a criterion to dissociate learning subspaces characteristic of fast (prompt) and slow (thermal and reburning) NO chemistry in both premixed and nonpremixed flame archetypes. For premixed flames a spatial length scale $\delta_{F F}$ is defined to separate the computational domain in a flame front region and a post-flame one. A corresponding mixing time scale $a_{F F}$ is identified for non-premixed flames. 


\section{A.1. Criterion definition}

In the premixed flamelet database, for each fresh gas equivalence ratio $\phi^{0}$, targeted thermo-chemical variables $\varphi$ such as the temperature and the species mass fractions are expressed in terms of the spatial coordinate $x$, that correspond to the direction normal to the flame front. $\varphi$ therefore reads:

$$
\varphi=\varphi_{p}\left(\phi^{0}, x_{p}\right)
$$

where $p$ subscript denotes solutions of premixed flame configurations.

Examples of NO mass fractions profiles are plotted in Fig. A1 for three different fresh gas equivalence ratio values (the corresponding mixture fraction value is also indicated in the figure). The transition between fast NO (in the flame front) and slow NO (in the post-flame) kinetics affects the second order material derivative of NO mass fraction, related to the second order derivative of $Y_{N O}$ in steady state, as follows:

$$
\frac{D^{2} Y_{N O}}{D t^{2}}=u^{2} \frac{d^{2} Y_{N O}}{d x_{p}^{2}}=\gamma\left(x_{p}\right)
$$

where $u$ is the flow velocity. The dimensionless $\gamma$ and the dimensionless $Y_{N O}$ second derivative are defined as follows:

$$
\gamma^{+}=\frac{\left|\gamma\left(x_{p}\right)\right|}{\max \left|\gamma\left(x_{p}\right)\right|} ; \quad \eta^{+}=\frac{\left|\frac{\mathrm{d}^{2} Y_{N O}\left(x_{p}\right)}{\mathrm{d} x_{p}^{2}}\right|}{\max \left(\left|\frac{\mathrm{d}^{2} Y_{N O}}{\mathrm{~d} x_{p}^{2}}\right|\right)}
$$

Figure A1 also plots $\gamma^{+}$and $\eta^{+}$in the three premixed $\mathrm{CH}_{4}$ /air flames previously introduced. The transition between flame front and post-flame NO formation is identified using the dimensionless absolute value of the second derivative $\eta^{+}$.

When $\eta^{+}$becomes lower than a certain threshold on the post-flame side the transition between fast and slow NO formation phenomena is defined at the spatial location $x_{p}$ which satisfy the following condition:

$$
\eta^{+}=\varepsilon
$$

where $\varepsilon$ is a user-defined threshold value. Equation A4 admits an ensemble of solutions $\left\{x_{1}^{p}, \ldots, x_{n}^{p}\right\}$. Among this ensemble of solutions, the flame front NO reaction layer $\delta_{F F}$ is defined so that fast NO chemistry is completed within the interval $\left[-\infty, \delta_{F F}\right]$ :

$$
\delta_{F F}\left(\phi^{0}\right)=\max \left\{x_{p_{1}}, \ldots, x_{p_{n}}\right\}
$$

where $x_{p}=0$ represents the beginning of the flame, defined here as the location where the flame temperature $T$ rises $10 K$ with respect to the fresh gases temperature $T^{f g}$. For premixed flame configurations, the flame front region and the post-flame one corresponds to the $1-\mathrm{D}$ domains $\mathcal{A}^{P} \in\left[-\infty, \delta_{F F}\right]$ and $\mathcal{A}^{P} \in\left[\delta_{F F}, \infty,\right]$, respectively. 

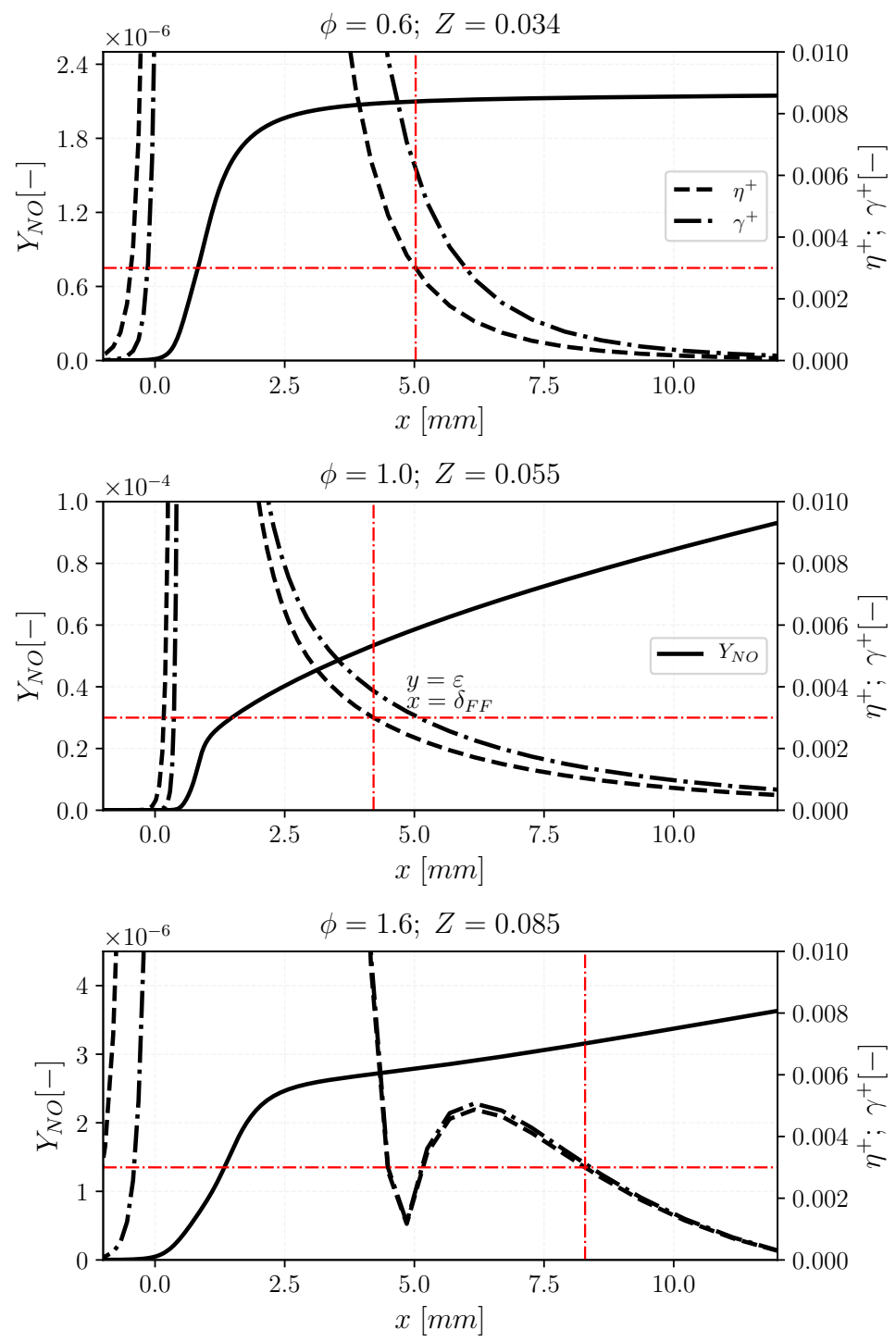

Figure A1.: NO mass fraction and the dimensionless $\eta^{+}$and $\gamma^{+}$quantities from detailed chemistry computations for different equivalence ratio values. The corresponding mixture fraction $Z$ is also indicated for each flame. 


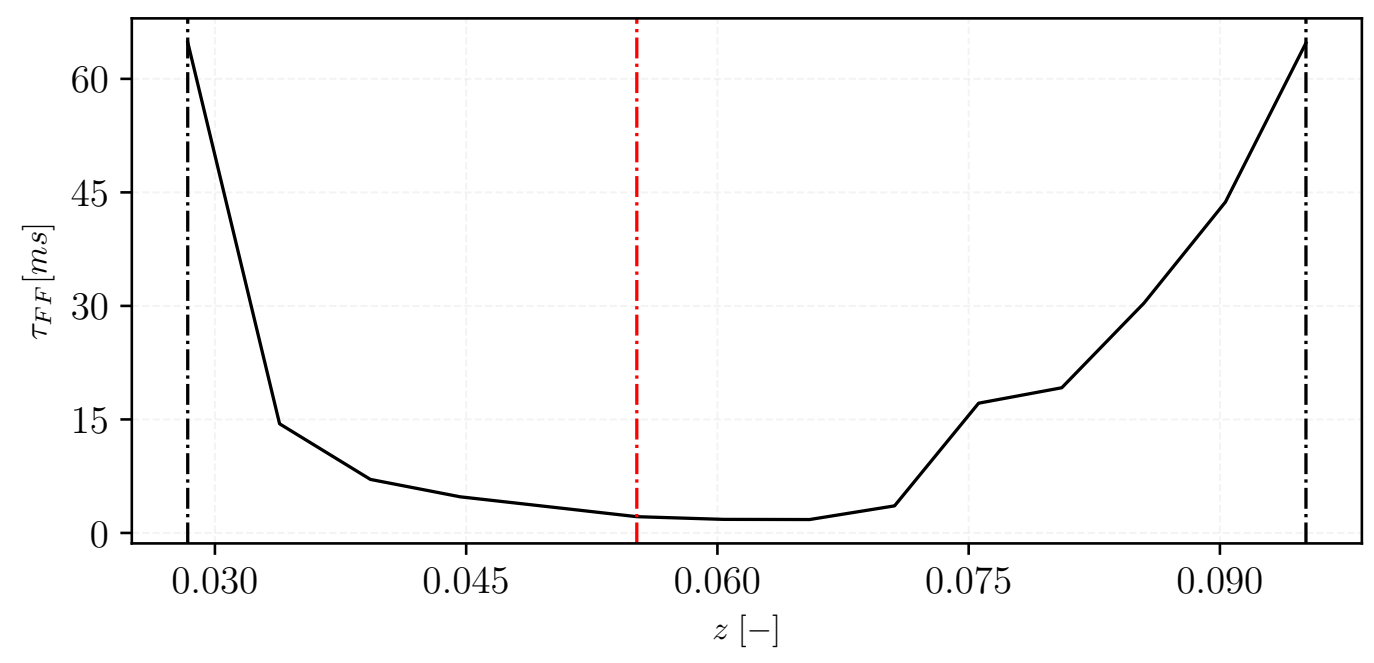

Figure A2.: Flame front residence time as function of mixture fraction. The stoichiometric mixture fraction, lean and rich flammability limits are also shown through the three vertical lines.

A cut-off time scale $\tau_{F F}$ between fast and slow NO chemistry is also introduced as the Lagrangian residence time taken by the fresh gases to reach $\delta_{F F}$ in premixed flames. $\tau_{F F}$ is defined as follows:

$$
\tau_{F F}\left(\phi^{0}\right)=\int_{0}^{\delta_{F F}\left(\phi^{0}\right)} \frac{1}{u_{p}\left(\phi^{0}\right)} d x
$$

A mixture fraction $Z$ is uniquely defined from the fresh gases equivalence ratio $\phi^{0}$ to reach 0 in pure air and 1 in pure fuel. $\tau_{F F}$ is plotted in Fig. A2 as function of the mixture fraction $Z$ in the whole flammability limit. The cut-off time scale averaged over the whole flammability domain reads:

$$
\bar{\tau}_{F F}=\frac{1}{\left(Z^{r}-Z^{l}\right)} \int_{Z_{l}}^{Z_{r}} \tau_{F F}(Z) d Z
$$

where $Z^{l}$ and $Z^{r}$ are values of mixture fraction at the lean and rich flammability limits, respectively.

\section{A.2. Extension to non-premixed counterflow flames}

To identify the non-premixed flames that have to be included in the flame front optimization database, the averaged cut-off time scale $\bar{\tau}_{F F}$, previously defined, is used. It is assumed that the transition time scale between fast and slow NO chemistry is of the same order of magnitude for premixed and non-premixed flames. As consequence, $\bar{\tau}_{F F}$ is here employed to estimate the critical strain rate, characteristic of non-premixed counterflow flames using the following simplified relation: 
Table B1.: Virtual NO sub-mechanisms kinetic rate parameters. Units are: cm, s, cal, mol and $\mathrm{K}$

\begin{tabular}{cccccc}
\hline Reaction & $A_{i}^{N O}$ & $E_{a}^{N O, i}$ & $\beta_{T}^{N O, i}$ & $F_{k}^{N O, i}$ & $\alpha_{k}^{N O, i}$ \\
\hline \hline & & & & $F_{F}^{N O, 1}=1.71$ & $\alpha_{V_{1}}^{N O, 1}=0.14$ \\
& & & & $\alpha_{V_{2}}^{N O, 1}=0.76$ \\
$R_{1}^{N O}$ & $1.5 \times 10^{18}$ & $3.5 \times 10^{4}$ & - & $F_{O x}^{N O, 1}=0.87$ & $\alpha_{V_{3}}^{N O, 1}=0.10$ \\
\hline & & & & & $\alpha_{N O, 2}^{N O, 2}=0.020$ \\
$R_{2}^{N O}$ & $1.9 \times 10^{24}$ & $7.6 \times 10^{4}$ & -0.08 & $F_{V_{1}}^{N O, 2}=1.24$ & $\alpha_{V_{2}}^{N O, 2}=0.98$ \\
\hline$R_{3}^{N O}$ & $1.1 \times 10^{18}$ & $4.7 \times 10^{3}$ & - & $F_{N O}^{N O, 3}=3.34$ & - \\
\hline$R_{4}^{N O}$ & $5.5 \times 10^{25}$ & $2.4 \times 10^{5}$ & - & $F_{V_{3}}^{N O, 4}=1.07$ & - \\
\hline$R_{5}^{N O}$ & $4.4 \times 10^{17}$ & $1.2 \times 10^{5}$ & - & $F_{V_{3}}^{N O, 5}=1.76$ & - \\
\hline & & & & $F_{N O, 6}^{N O, 6}=0.19$ & \\
$R_{6}^{N O}$ & $3.6 \times 10^{21}$ & $2.2 \times 10^{5}$ & - & $F_{V_{2}}^{N O, 6}=0.97$ & - \\
\hline
\end{tabular}

$$
a_{F F}=1 / \bar{\tau}_{F F} .
$$

All flamelets computed with a strain rate higher than $a_{F F}\left(\mathcal{A}^{N P} \in\left[a_{F F}, a_{q}\right]\right)$ will serve as a target for optimizing the flame front chemistry block (reactions $R_{1}^{N O}-R_{3}^{N O}$ ).

\section{Appendix B. NO sub-mechanism for $\mathrm{CH}_{4} / \mathrm{Air}$}

Table B1 gives the optimized kinetic rate parameters for the NO-virtual submechanism: Fot pre-exponential constants that are mixture-fraction dependent the value is reported at stoichiometry.

The correction functions $f_{i}=A_{i}(Z) / A_{i}^{s t}$ applied to the pre-exponential constants $A_{2}, A_{4}, A_{5}$ and $A_{6}$ are plotted as function of the mixture fraction in Fig. B1.

\section{References}

\section{References}

[1] M. Cailler, N. Darabiha, and B. Fiorina, Development of a virtual optimized chemistry method. Application to hydrocarbon/air combustion, Combustion and Flame 

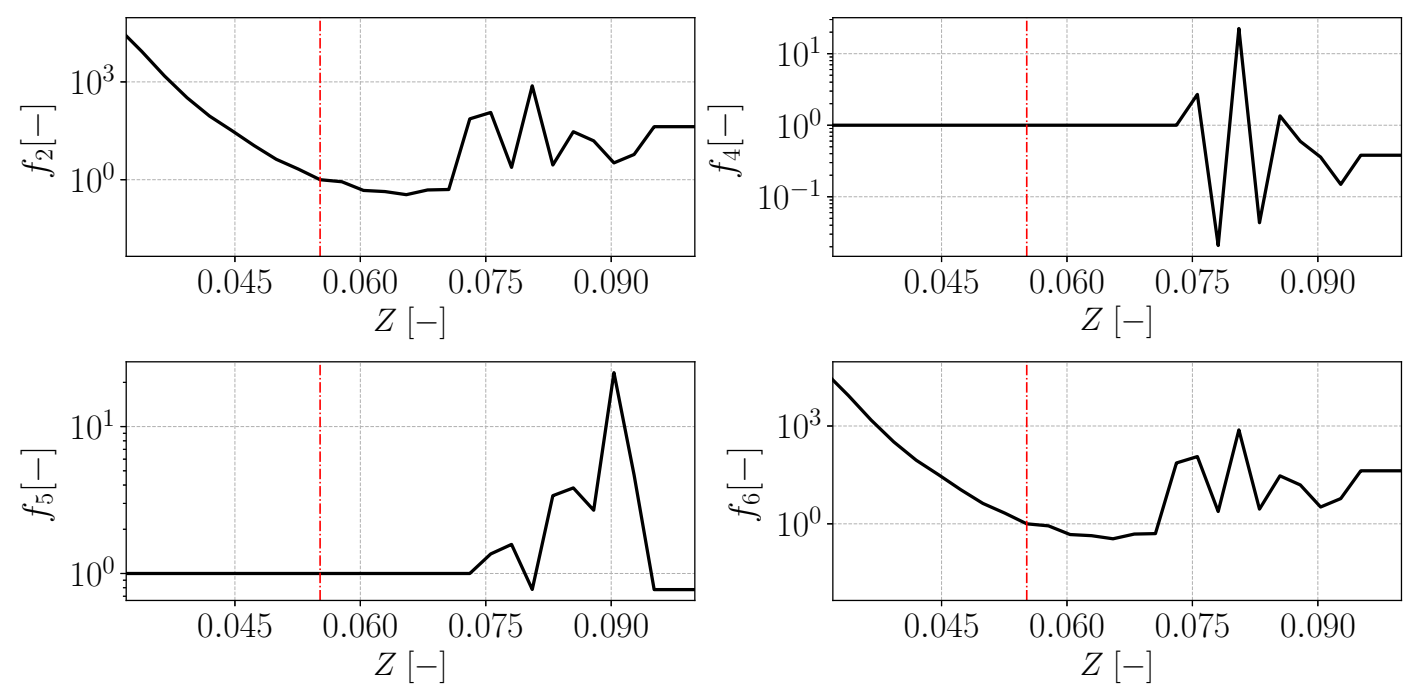

Figure B1.: Correction function $f_{i}$ applied to pre-exponential constants $A_{2}, A_{4}, A_{5}$ and $A_{6}$, respectively. The stoichiometric mixture fraction is also shown through the vertical line.

211 (2020), pp. 281-302.

[2] I.E.A. International Energy Agency, World energy outlook 2016, https://webstore.iea.org/world-energy-outlook-2016 (2016).

[3] G.P. Smith, D.M. Golden, M. Frenklach, B. Eiteener, M. Goldenberg, C.T. Bowman, R.K. Hanson, W.C. Gardiner, V.V. Lissianski, and Z.W. Qin, http://www.me.berkeley.edu/gri_mech (2011).

[4] A.H. Lefebvre, Gas turbine combustion, CRC press, 1998.

[5] N. Darabiha, Transient behaviour of laminar counterflow hydrogen-air diffusion flames with complex chemistry, Combust. Sci. Techol. 86 (1992), pp. 163-181.

[6] C. Fenimore, Formation of nitric oxide in premixed hydrocarbon flames, in Symposium (International) on Combustion, Vol. 13. Elsevier, 1971, pp. 373-380.

[7] A. Hayhurst and I. Vince, Nitric oxide formation from $N_{2}$ in flames: the importance of "prompt" NO, Progress in Energy and Combustion Science 6 (1980), pp. $35-51$.

[8] Y.B. Zeldovich, Selected Works of Yakov Borisovich Zeldovich, Volume I: Chemical Physics and Hydrodynamics, Vol. 140, Princeton University Press, 2014.

[9] K.K. Kuo, Principles of combustion, John Wiley 2005, 2005.

[10] T. Faravelli, A. Frassoldati, and E. Ranzi, Kinetic modeling of the interactions between NO and hydrocarbons in the oxidation of hydrocarbons at low temperatures, Combustion and Flame 132 (2003), pp. 188-207.

[11] A. Frassoldati, T. Faravelli, and E. Ranzi, Kinetic modeling of the interactions between NO and hydrocarbons at high temperature, Combustion and Flame 135 (2003), pp. 97-112.

[12] P. Glarborg, J.A. Miller, B. Ruscic, and S.J. Klippenstein, Modeling nitrogen chemistry in combustion, Progress in Energy and Combustion Science 67 (2018), pp. 31-68.

[13] T. Lu and C.K. Law, Toward accommodating realistic fuel chemistry in largescale computations, Progress in Energy and Combustion Science 35 (2009), pp. $192-215$. 
[14] H. Wang and M. Frenklach, Detailed reduction of reaction mechanisms for flame modeling, Combustion and Flame 87 (1991), pp. 365-370.

[15] B. Fiorina, D. Veynante, and S. Candel, Modeling combustion chemistry in large eddy simulation of turbulent flames, Flow, Turbulence and Combustion 94 (2015), pp. $3-42$.

[16] B. Fiorina and M. Cailler, Accounting for complex chemistry in the simulations of future turbulent combustion systems, in AIAA Scitech 2019 Forum. 2019, p. 0995.

[17] C.K. Westbrook and F.L. Dryer, Simplified reaction mechanisms for the oxidation of hydrocarbon fuels in flames, Combustion science and technology 27 (1981), pp. $31-43$.

[18] W. Jones and R. Lindstedt, Global reaction schemes for hydrocarbon combustion, Combustion and flame 73 (1988), pp. 233-249.

[19] N. Peters, Laminar diffusion flamelet models in non-premixed turbulent combustion, Progress in energy and combustion science 10 (1984), pp. 319-339.

[20] O. Gicquel, N. Darabiha, and D. Thévenin, Laminar premixed hydrogen/air counterflow flame simulations using flame prolongation of ILDM with differential diffusion, Proceedings of the Combustion Institute 28 (2000), pp. 1901-1908.

[21] J. Van Oijen, F. Lammers, and L. De Goey, Modeling of complex premixed burner systems by using flamelet-generated manifolds, Combustion and Flame 127 (2001), pp. 2124-2134.

[22] T. Lu and C.K. Law, A criterion based on computational singular perturbation for the identification of quasi steady state species: A reduced mechanism for methane oxidation with NO chemistry, Combustion and Flame 154 (2008), pp. 761-774.

[23] P. Pepiot-Desjardins and H. Pitsch, An efficient error-propagation-based reduction method for large chemical kinetic mechanisms, Combustion and Flame 154 (2008), pp. $67-81$.

[24] E. Fernandez-Tarrazo, A.L. Sánchez, A. Linan, and F.A. Williams, A simple onestep chemistry model for partially premixed hydrocarbon combustion, Combustion and Flame 147 (2006), pp. 32-38.

[25] B. Franzelli, E. Riber, M. Sanjosé, and T. Poinsot, A two-step chemical scheme for kerosene-air premixed flames, Combustion and Flame 157 (2010), pp. 1364-1373.

[26] T. Jaravel, E. Riber, B. Cuenot, and G. Bulat, Large eddy simulation of an industrial gas turbine combustor using reduced chemistry with accurate pollutant prediction, Proceedings of the Combustion Institute 36 (2017), pp. 3817-3825.

[27] T. Jaravel, E. Riber, B. Cuenot, and P. Pepiot, Prediction of flame structure and pollutant formation of Sandia flame $D$ using large eddy simulation with direct integration of chemical kinetics, Combustion and Flame 188 (2018), pp. 180-198.

[28] U. Maas and S.B. Pope, Implementation of simplified chemical kinetics based on intrinsic low-dimensional manifolds, in Symposium (International) on Combustion, Vol. 24. Elsevier, 1992, pp. 103-112.

[29] V. Bykov and U. Maas, The extension of the ildm concept to reaction-diffusion manifolds, Combustion Theory and Modelling 11 (2007), pp. 839-862.

[30] J. Nafe and U. Maas, Modeling of NO formation based on ildm reduced chemistry, Proceedings of the Combustion Institute 29 (2002), pp. 1379-1385.

[31] G. Godel, P. Domingo, and L. Vervisch, Tabulation of NOx chemistry for largeeddy simulation of non-premixed turbulent flames, Proceedings of the Combustion Institute 32 (2009), pp. 1555-1561.

[32] A. Vreman, B. Albrecht, J. Van Oijen, L. De Goey, and R. Bastiaans, Premixed and nonpremixed generated manifolds in large-eddy simulation of Sandia flame $D$ 
and F, Combustion and Flame 153 (2008), pp. 394-416.

[33] M. Ihme and H. Pitsch, Modeling of radiation and nitric oxide formation in turbulent nonpremixed flames using a flamelet/progress variable formulation, Physics of Fluids 20 (2008), p. 055110.

[34] A. Ketelheun, C. Olbricht, F. Hahn, and J. Janicka, No prediction in turbulent flames using LES/FGM with additional transport equations, Proceedings of the Combustion Institute 33 (2011), pp. 2975-2982.

[35] F. Pecquery, V. Moureau, G. Lartigue, L. Vervisch, and A. Roux, Modelling nitrogen oxide emissions in turbulent flames with air dilution: Application to LES of a non-premixed jet-flame, Combustion and Flame 161 (2014), pp. 496-509.

[36] P.E. Vervisch, O. Colin, J.B. Michel, and N. Darabiha, NO relaxation approach (NORA) to predict thermal NO in combustion chambers, Combustion and Flame 158 (2011), pp. 1480-1490.

[37] B. Fiorina, O. Gicquel, L. Vervisch, S. Carpentier, and N. Darabiha, Approximating the chemical structure of partially premixed and diffusion counterflow flames using FPI flamelet tabulation, Combustion and flame 140 (2005), pp. 147-160.

[38] P.D. Nguyen, L. Vervisch, V. Subramanian, and P. Domingo, Multidimensional flamelet-generated manifolds for partially premixed combustion, Combustion and Flame 157 (2010), pp. 43-61.

[39] V. Bykov and U. Maas, Problem adapted reduced models based on reactiondiffusion manifolds (REDIMs), Proceedings of the Combustion Institute 32 (2009), pp. 561-568.

[40] M. Cailler, N. Darabiha, D. Veynante, and B. Fiorina, Building-up virtual optimized mechanism for flame modeling, Proc. Combust. Inst. (2017).

[41] G. Maio, M. Cailler, R. Mercier, and B. Fiorina, Virtual chemistry for temperature and $C O$ prediction in LES of non-adiabatic turbulent flames, Proceedings of the Combustion Institute 37 (2019), pp. 2591-2599.

[42] A. Cuoci, A. Frassoldati, T. Faravelli, and E. Ranzi, A computational tool for the detailed kinetic modeling of laminar flames: Application to $\mathrm{C}_{2} \mathrm{H}_{4} / \mathrm{CH}_{4}$ coflow flames, Combustion and Flame 160 (2013), pp. 870-886.

[43] J. Caudal, B. Fiorina, B. Lab??gorre, and O. Gicquel, Modeling interactions between chemistry and turbulence for simulations of partial oxidation processes, Fuel Processing Technology 134 (2015), pp. 231-242, Available at http://dx. doi.org/10.1016/j.fuproc.2015.01.040.

[44] OpenFOAM (2019). Available at www.openfoam.org.

[45] A. Cuoci, A. Frassoldati, T. Faravelli, and E. Ranzi, Numerical modeling of laminar flames with detailed kinetics based on the operator-splitting method, Energy \& Fuels 27 (2013), pp. 7730-7753.

[46] A. Stagni, A. Cuoci, A. Frassoldati, T. Faravelli, and E. Ranzi, Lumping and reduction of detailed kinetic schemes: an effective coupling, Industrial \& Engineering Chemistry Research 53 (2013), pp. 9004-9016.

[47] M.S. Day and J.B. Bell, Numerical simulation of laminar reacting flows with complex chemistry, Combustion Theory and Modelling 4 (2000), pp. 535-556.

[48] C.K. Law, Combustion at a crossroads: Status and prospects, Proceedings of the Combustion Institute 31 (2007), pp. 1-29.

[49] P. Pepiot, Automatic strategies to model transportation fuel surrogates, Stanford University Stanford, CA, Ph.D thesis, 2008. 